\title{
Stellar streams in chameleon gravity
}

\author{
A. P. Naik $\odot^{*}$ and N.W. Evans ${ }^{\dagger}$ \\ Institute of Astronomy, University of Cambridge, Madingley Road, Cambridge CB3 OHA, United Kingdom \\ E. Puchwein \\ Leibniz-Institut für Astrophysik Potsdam, An der Sternwarte 16, 14482 Potsdam, Germany \\ H. Zhao \\ Kavli Institute for Cosmology Cambridge, Madingley Road, Cambridge CB3 OHA, United Kingdom \\ and Scottish Universities Physics Alliance, University of St. Andrews, \\ North Haugh, St. Andrews, Fife KY16 9SS, United Kingdom
}

A.-C. Davis

Department of Applied Mathematics and Theoretical Physics, Centre for Mathematical Sciences, Cambridge CB2 OWA, United Kingdom

(Received 10 February 2020; accepted 24 September 2020; published 27 October 2020)

Theories of gravity that incorporate new scalar degrees of freedom typically require "screening mechanisms" to ensure consistency with Solar System tests. One widely studied mechanism-the chameleon mechanism — can lead to violations of the equivalence principle (EP), as screened and unscreened objects fall differently. If the stars are screened but the surrounding dark matter is not, EP violation can lead to asymmetry between leading and trailing streams from tidally disrupted dwarf galaxies in the Milky Way halo. We provide analytic estimates of the magnitude of this effect for realistic Galactic mass distributions, demonstrating that it is an even more sensitive probe than suggested previously. Using a restricted $N$-body code, we simulate four satellites with a range of masses and orbits, together with a variety of strengths of the fifth force and screening levels of the Milky Way and satellite. The ratio of the cumulative number function of stars in the leading and trailing stream as a function of longitude from the satellite is computable from simulations and measurable from the stellar data and can provide a direct test of chameleon gravity. We forecast constraints for streams at large Galactocentric distances, which probe deeper into chameleon parameter space, using the specific example case of Hu-Sawicki $f(R)$ gravity. Streams in the outer reaches of the Milky Way halo (with apocenters between 100 and $200 \mathrm{kpc}$ ) provide easily attainable constraints at the level of $\left|f_{R 0}\right|=10^{-7}$. Still more stringent constraints at the level of $10^{-7.5}$ or even $10^{-8}$ are plausible provided the environmental screening of the satellite is accounted for, and screening of the Milky Way's outer halo by the Local Group is not yet triggered in this range. These would be among the tightest astrophysical constraints to date. We note three further signatures of chameleon gravity: (i) the trailing stellar stream may become detached from the dark matter progenitor if all the stars are lost, (ii) in the extreme fifth force regime, striations in the stellar trailing tail may develop from material liberated at successive pericentric passages, and (iii) if the satellite is fully screened, its orbital frequency is lower than that of the associated dark matter, which is preferentially liberated into the leading tidal tail.

DOI: 10.1103/PhysRevD.102.084066

\section{INTRODUCTION}

Stellar streams and substructures are the wreckage of dwarf galaxies and globular clusters that have fallen into and are being torn apart by the Milky Way's tidal field. In the past, such substructures have usually been identified as

\footnotetext{
*an485@ast.cam.ac.uk †nwe@ast.cam.ac.uk

"th4@st-andrews.ac.uk
}

overdensities of resolved stars, as in the "field of streams" image from the Sloan Digital Sky Survey [1]. There, using multiband photometry, the stellar halo of the Milky Way was revealed as being composed of criss-crossing stellar streams, the detritus of satellite galaxies. However, streams and substructure remain kinematically cold and so identifiable in phase space long after they have ceased to be recognizable in star counts against the stellar background of the Galaxy [2]. The debris persists for a large fraction of a Hubble time, sometimes longer, so substructures in phase 
space remain to the present day. Searches in phase space for streams are much more powerful than searches in configuration space.

The Gaia satellite is a scanning satellite of the European Space Agency that is monitoring all objects brighter than magnitude $G \approx 20$ around 70 times over a period of $5 \mathrm{yr}$ (though the mission lifetime has recently been extended) $[3,4]$. Its telescopes are providing magnitudes, parallaxes, proper motions and broadband colors for over a billion stars in the Galaxy ( $\approx 1 \%$ of the Milky Way stellar population) within the Gaia-sphere-or within roughly $20 \mathrm{kpc}$ of the Sun for main sequence stars, $100 \mathrm{kpc}$ for giants. We now possess detailed phase space information, often with spectroscopic and chemical data from cross-matches with other surveys. This has led to the discovery of abundant streams and substructures [5-8]. Streams discovered by Gaia are already being followed up spectroscopically to give six-dimensional (6D) phase space data [9]. Bright tracers such as blue horizontal branch stars or RR Lyraes can be seen out to distances of $250 \mathrm{kpc}$, assuming Gaia's limiting magnitude of G 20.5. Stars near the tip of the red giant branch can be seen even further out to at least $600 \mathrm{kpc}$. In future, this should enable Gaia to provide astrometry for very distant streams, perhaps beyond the edge of the Milky Way's dark halo.

If a stream were a simple orbit, then the positions and velocities of stars would permit the acceleration and force field to be derived directly from the 6D data. Streams are more complex than orbits $[10,11]$, but the principle remains the same- their evolution constrains the matter distribution and theory of gravity. Although this idea has been in the literature for some years, exploitation has been sparse primarily because of the limited number of streams with 6D data before Gaia. This field is therefore ripe for further exploitation in the Gaia era.

Because of their different ages and different positions in phase space, different streams may tell us different things about the theory of gravity. For example, Thomas et al. [12] show that streams from globular clusters are lopsided in modified Newtonian dynamics because the "external field effect" violates the strong equivalence principle. Meanwhile, Kesden and Kamionkowski $[13,14]$ demonstrated that if a so-called "fifth force" couples to dark matter but not to baryons, this violation of the equivalence principle (EP) leads to large, observable asymmetries in stellar streams from dark-matter-dominated dwarf galaxies. Specifically, the preponderance of stars are disrupted via the outer Lagrange point rather than the inner one, and the trailing stream is consequently significantly more populated than the leading one. Building on that work, Keselman, Nusser, and Peebles [15] explored the regime of fifth forces much stronger than those investigated by Kesden and Kamionkowski and found a number of interesting results, including plausible formation scenarios for the Sagittarius stream, the Draco satellite, and progenitorless "orphan" streams around the Milky Way.
In the intervening years since the work of Kesden and Kamionkowski, screened modified gravity theories have become the subject of increasing attention [16-19]. In these theories, a scalar field coupled to gravity is introduced, giving rise to gravitational-strength fifth forces. For the field to retain cosmological relevance while also avoiding violations of stringent Solar System tests of gravity, screening mechanisms are introduced $[20,21]$. There are several varieties of screening mechanism, but in the one studied here-the chameleon mechanism-the mass of the scalar field is environment dependent, such that the fifth force is suppressed within deep potential wells [22]. In other words, in dense environments like our Solar System, the chameleon becomes invisible to fifth force searches, hence its name.

A widely studied class of modified gravity theories is $f(R)$ gravity [23]. Here, the Ricci scalar $R$ in the EinsteinHilbert action is generalized to $R+f(R)$. The Hu-Sawicki form of $f(R)$ [24] exhibits the chameleon mechanism and has been shown to be formally equivalent to a subclass of scalar-tensor theories of gravity [25]. The key parameter is the present-day cosmic background value of the scalar field, $f_{R 0}$. In the present work, we do not assume $\mathrm{Hu}-$ Sawicki $f(R)$ gravity but sometimes use the parameter $f_{R 0}$ as a concrete example to illustrate the possible constraints achievable from stellar streams, noting that constraints are also obtainable in the wider chameleon space.

A complete compendium of current constraints on $f(R)$ gravity and chameleon gravity more generally can be found in the review article by Burrage and Sakstein [26]. It is worth noting that some of the strongest constraints to date have come from weak-field astrophysical probes. Moreover, Baker et al. [27] identify a "desert" in modified gravity parameter space accessible only to galaxy-scale probes and have launched the "novel probes" project, aimed at connecting theorists with observers in order to devise tests to probe this region. Accordingly, several recent works [28-33] have studied imprints of screened modified gravity on galaxy scales.

In chameleon theories, main sequence stars will have sufficiently deep potential wells to self-screen against the fifth force. A diffuse dark matter or gaseous component of sufficiently low mass, however, will be unscreened. As a result, the EP is effectively violated, leading to a number of distinct signatures, as listed by Hui, Nicolis, and Stubbs [34]. Indeed, several of the galaxy-scale studies mentioned in the previous paragraph searched for signatures in this list, as well as other signatures of EP violation.

The present work explores the idea that effective EP violation of chameleon gravity should give rise to the stellar stream asymmetries predicted by Kesden and Kamionkowski $[13,14]$. We will show that tidal streams in the Milky Way, observable with Gaia, can provide constraints that are comparable to, or stronger than, other astrophysical probes. Section II gives a brief introduction to 
the fifth force in chameleon theories before providing a new calculation of the magnitude of the effect, extending the original work of Kesden and Kamionkowski [13]. Next, Sec. III describes the Milky Way and satellite models that we use in our simulation code, the methodology and validation of which are in turn described, respectively, in Secs. IV and V. Section VI describes results for a range of tidal streams, inspired by examples discovered recently in large photometric surveys or the Gaia datasets. Finally, Sec. VII gives some concluding remarks.

\section{THEORY}

\section{A. Chameleon fifth forces}

In scalar-tensor gravity theories, new scalar degrees of freedom in the gravitational sector couple to matter, giving rise to gravitational-strength fifth forces. Chameleon theories are a class of scalar-tensor theories in which these fifth forces are suppressed in regions of high density or deep gravitational potential [22]. In this section, we cover only the most salient aspects of chameleon theories and refer the reader elsewhere [26] for a more complete description of the formalism and summary of existing constraints.

Consider a spherical overdensity embedded within a region of average cosmic density. If the gravitational well of the object is sufficiently deep, a central region of radius $r_{\text {scr }}$ will be "screened," such that no fifth forces act within the region; $r_{\text {scr }}$ is the "screening radius" of the object. Outside the screening radius, an unscreened test particle will experience an acceleration due to the fifth force given by Eq. (3.6) of Ref. [26]:

$$
a_{5}(r)=2 \beta^{2} \frac{G\left(M(r)-M\left(r_{\mathrm{scr}}\right)\right)}{r^{2}},
$$

where $M(r)$ is the mass enclosed within radius $r$ and $\beta$ is the coupling strength. In other words, the fifth force is sourced only by the mass lying between the screening radius and the test particle. We have also assumed here that the Compton wavelength of the theory is much larger than the characteristic length scales of the system.

Equation (1) gives the fifth force experienced by an unscreened test particle outside the screening radius of an overdense object, but the situation is complicated further in the case where instead of a test particle, we have another extended object-for example, a star or dwarf galaxy situated outside the screening radius of its host galaxy. In this case, the acceleration of object $i$ (mass $M_{i}$, radius $r_{i}$, screening radius $\left.r_{\mathrm{scr}, i}\right)$ due to the fifth force is given by

$$
a_{5}(r)=2 \beta^{2} Q_{i} \frac{G\left(M(r)-M\left(r_{\mathrm{scr}}\right)\right)}{r^{2}},
$$

where $Q_{i}$ is the "scalar charge" of object $i$, given in turn by

$$
Q_{i}=\left(1-\frac{M_{i}\left(r_{\mathrm{scr}, i}\right)}{M_{i}}\right)
$$

Thus, the test object experiences the full fifth force only if it is fully unscreened (i.e., $r_{\text {scr }}=0$ ) and experiences no fifth force if it is fully screened $\left(r_{\mathrm{scr}}=r_{i}\right)$. In the intermediate case where the object is partially screened, it experiences a reduced fifth force. In this work, we assume stars to be fully self-screened (i.e., $Q=0$ ) and dark matter to be fully unscreened (i.e., $Q=1$ ). For the satellite galaxies (i.e., the stream progenitors), we explore a number of regimes, spanning fully screened, partially screened, and fully unscreened.

A commonly used parametrization of chameleon theories is in terms of the coupling strength $\beta$ and the "selfscreening parameter" $\chi_{c}$. The latter parameter determines which astrophysical objects are fully or partially screened and can be used to calculate their screening radii. Note that in the case of Hu-Sawicki $f(R)$ gravity, $\beta$ is fixed to $\sqrt{1 / 6}$, while $\chi_{c}=-f_{R 0}$.

In order to derive constraints in the $\beta / \chi_{c}$ plane or $f_{R 0}$ space from stellar streams around the Milky Way, we would need to adopt some prescription to convert $\chi_{c}$ to Milky Way and satellite screening radii. Analytical formulas exist in the case of an isolated spherical body [35,36], but such a treatment would neglect the environmental contribution of the Local Group to the Milky Way's screening, the environmental contribution of the Milky Way to the satellite's screening, and the impact of the nonsphericity of the Milky Way. The calculation therefore requires numerical methods in more realistic scenarios [28]. In this work, we instead use $\beta, r_{\mathrm{scr}, \mathrm{MW}}$, and $r_{\mathrm{scr}, \mathrm{sat}}$ as input parameters for reasons of computational ease. However, in Sec. VID, we investigate the connection between $f_{R 0}$ and $r_{\mathrm{scr}, \mathrm{MW}}$ in order to forecast constraints from future data.

\section{B. Stream asymmetries}

\section{A physical picture}

We begin with a physical picture of the cause of stream asymmetries. Consider a satellite represented by a point mass $m$. For the moment, let us neglect any fifth forces and assume that the Milky Way can also be represented as a point mass $M$, so both satellite and the Milky Way are moving on circular orbits with frequency $\Omega$ around their common center of mass.

We use a coordinate system whose origin is at the center of the satellite. Then, a star at position $\mathbf{r}_{\mathrm{s}}$ moves in an "effective" gravitational potential given by (e.g., [37,38])

$$
\Phi_{\mathrm{eff}}\left(\mathbf{r}_{\mathrm{s}}\right)=-\frac{G m}{r_{\mathrm{s}}}-\frac{G M}{\left|\mathbf{r}_{h}-\mathbf{r}_{\mathrm{s}}\right|}-\frac{1}{2} \Omega^{2}\left|\mathbf{r}_{\mathrm{s}}-\mathbf{r}_{\mathrm{cm}}\right|^{2},
$$

where $\mathbf{r}_{\mathrm{h}}$ is the position of the point mass representing the Milky Way and $\mathbf{r}_{\mathrm{cm}}$ is the position of the center of mass. 
We use the convention $r_{\mathrm{s}}=\left|\mathbf{r}_{\mathrm{s}}\right|$ to denote the modulus of any vector. The first two terms are the gravitational potentials of the satellite and Milky Way, respectively, while the final term provides the centrifugal force due to the frame of reference, which is rotating about the center of mass with frequency $\Omega$ :

$$
\Omega=\sqrt{\frac{G(M+m)}{r_{\mathrm{h}}{ }^{3}}} .
$$

In practice, the mass of a typical satellite $m$ is at least 2 orders of magnitude less than the mass of the Milky Way, and so its contribution to the frequency can be neglected.

The stationary points of the effective potential $\Phi_{\text {eff }}$ are the Lagrange points or equilibria at which the net force on a star at rest vanishes. In the circular restricted three-body problem, there are five Lagrange points. Matter is pulled out of the satellite at the "L1" and "L2" saddle points, henceforth the "inner" and "outer" Lagrange points, respectively. These are situated either side of the satellite, collinear with the satellite and Milky Way. Leading (trailing) streams originate at the inner (outer) Lagrange points, which lie at (see Sec. VIII 31 of Binney and Tremaine [38])

$$
r_{\mathrm{t}} \approx\left(\frac{m}{3 M}\right)^{1 / 3} r_{h}
$$

with respect to the satellite center.
Now consider how the system behaves if a fifth force acts on the dark matter. Neglecting any screening, and assuming the satellite is dark matter dominated, the orbit will circle more quickly with frequency given by

$$
\Omega^{\prime}=\sqrt{\frac{G^{\prime}(M+m)}{r_{h}{ }^{3}}} \approx \sqrt{\frac{G^{\prime} M}{r_{h}{ }^{3}}},
$$

where $G^{\prime} \equiv\left(1+2 \beta^{2}\right) G$. The effective potential experienced by a dark matter particle in this system is

$\Phi_{\text {eff }, \mathrm{DM}}\left(\mathbf{r}_{s}\right)=-\frac{G^{\prime} m}{r_{s}}-\frac{G^{\prime} M}{\left|\mathbf{r}_{h}-\mathbf{r}_{s}\right|}-\frac{1}{2} \Omega^{\prime 2}\left|\mathbf{r}_{s}-\mathbf{r}_{\mathrm{cm}}\right|^{2}$.

This is tantamount to a linear rescaling of Eq. (4), and the locations of the critical points are therefore unchanged relative to the standard gravity case. However, the effective potential is different for a star which does not feel the fifth force, namely

$$
\Phi_{\mathrm{eff}, *}\left(\mathbf{r}_{s}\right)=-\frac{G m}{r_{s}}-\frac{G M}{\left|\mathbf{r}_{h}-\mathbf{r}_{s}\right|}-\frac{1}{2} \Omega^{\prime 2}\left|\mathbf{r}_{s}-\mathbf{r}_{\mathrm{cm}}\right|^{2} .
$$

This is not a linear multiple of Eq. (4), and the locations of the Lagrange points are consequently altered. The two panels of Fig. 1 shows contour maps of the effective potentials for dark matter and stars, for $M=10^{12} M_{\odot}$,

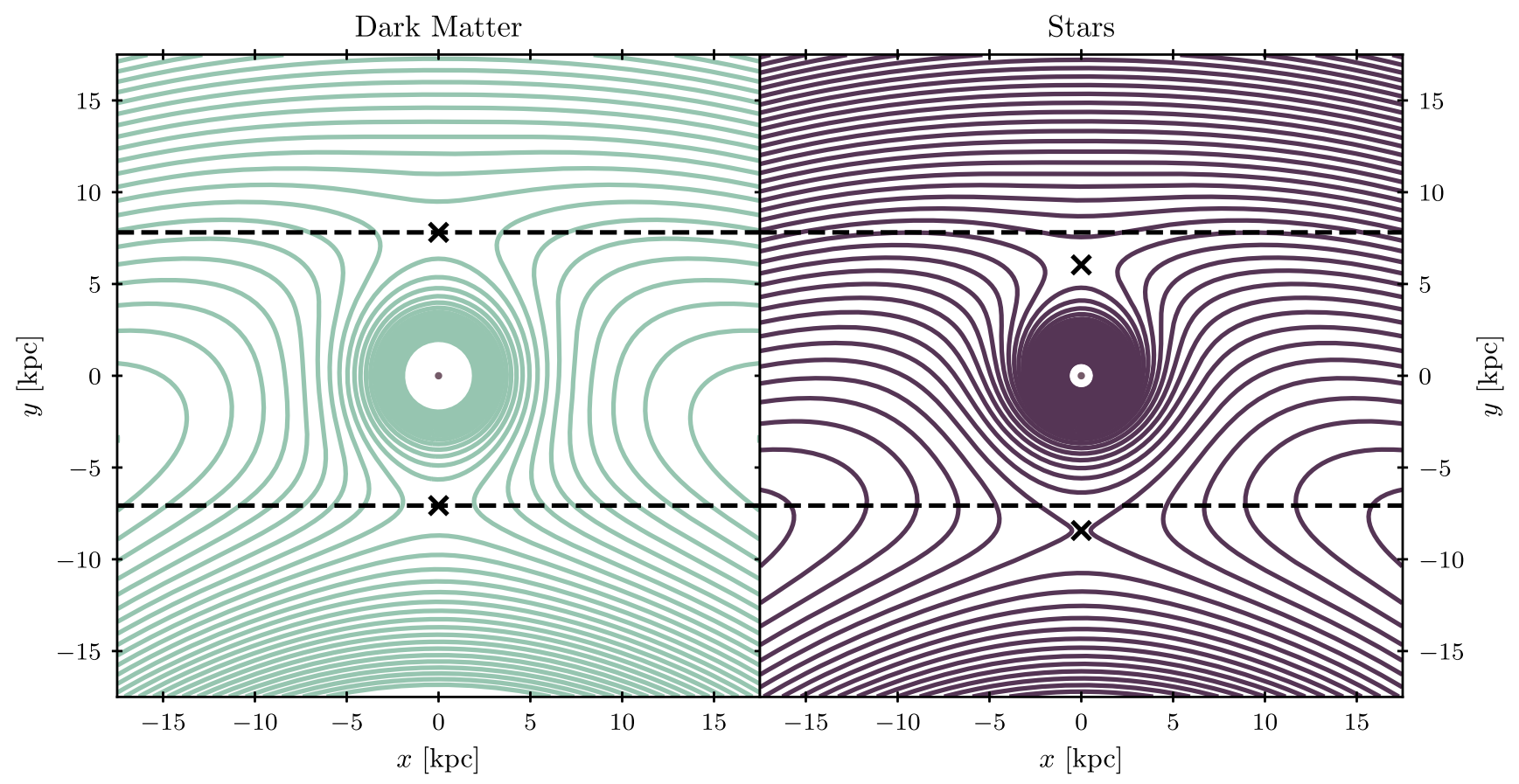

FIG. 1. Left: Contour map of the effective potential for the dark matter $\Phi_{\text {eff,DM }}$ as given by Eq. (8). Right: Contour map of the effective potential for the stars $\Phi_{\mathrm{eff}, *}$ as given by Eq. (9). In both panels, the satellite is at the origin and the Galactic center is at $(0,-50,0) \mathrm{kpc}$. The inner and outer Lagrange points are marked by crosses. To guide the eye, black dashed lines marking the positions of the DM Lagrange points span the figure. The asymmetry of the Lagrange points for the stellar effective potential illustrates the cause of the stream asymmetries under chameleon gravity. (Parameters: $M=10^{12} M_{\odot}, m=10^{10} M_{\odot}$, and $\beta=0.5$.) 
$m=10^{10} M_{\odot}, r_{h}=50 \mathrm{kpc}$, and $\beta=0.5$. Also indicated on the diagram are the locations of the inner and outer Lagrange points of the potentials.

In the dark matter case, the points are approximately equidistant from the satellite center. However, a significant asymmetry is visible in the stellar effective potential, with the outer Lagrange point being much closer to the satellite and at a lower effective potential. Thus, stars are much more likely to be stripped from the satellite at the outer Lagrange point, and the trailing stream will consequently be more populated than the leading one.

Physically, we can understand this effect in terms of force balance. The stars are being dragged along by the satellite, which is orbiting at an enhanced rotation speed due to the fifth force. This enhanced speed means that the outward centrifugal force on the stars is greater than the inward gravitational attraction by the Milky Way. The consequence of this net outward force is that stars can be stripped from the satellite more easily if they are at larger Galactocentric radii than the satellite and less easily if they are at smaller radii. This is reflected in the positions of the Lagrange points.

Stars unbound from the satellite will be on a slower orbit around the Milky Way than their progenitor. If $\beta$ is sufficiently large, then stars that are initially in the leading stream can fall behind and end in the trailing stream.

\section{Circular restricted three-body problem}

We now solve for the stream asymmetries in the circular restricted three-body problem, following and correcting Ref. [13]. This is a useful preliminary before passing to the general case. In Newtonian gravity, the forces balance at the inner and outer Lagrange points, and so

$$
\begin{aligned}
& -\frac{G M}{\left(r_{h}-r_{t}\right)^{2}}+\frac{G m}{r_{t}^{2}}+\frac{G(M+m)}{r_{h}{ }^{3}}\left(\frac{M r_{h}}{M+m}-r_{t}\right)=0, \\
& -\frac{G M}{\left(r_{h}+r_{t}\right)^{2}}-\frac{G m}{r_{t}^{2}}+\frac{G(M+m)}{r_{h}{ }^{3}}\left(\frac{M r_{h}}{M+m}+r_{t}\right)=0 .
\end{aligned}
$$

We recall that the inertial frame is rotating about the center of mass, and so the centrifugal terms in Eqs. (10) and (11) depend on the distance of the Lagrange point to the center of mass, not the Galactic center [cf. Eqs. (14) and (15) of Ref. [13] ].

We now define $u=r_{t} / r_{h}$ and $u^{\prime}=r_{t}^{\prime} / r_{h}$ for the inner and outer Lagrange points, respectively, and obtain

$$
\begin{aligned}
u^{3} & =\frac{m}{M} \frac{\left(1-u^{3}\right)(1-u)^{2}}{3-3 u+u^{2}}, \\
u^{\prime 3} & =\frac{m}{M} \frac{\left(1-u^{\prime 3}\right)\left(1+u^{\prime}\right)^{2}}{3+3 u^{\prime}+u^{\prime 2}} .
\end{aligned}
$$

Solving, we find that

$$
\begin{aligned}
& u \approx\left(\frac{m}{3 M}\right)^{1 / 3}\left(1-\frac{u}{3}\right), \\
& u^{\prime} \approx\left(\frac{m}{3 M}\right)^{1 / 3}\left(1+\frac{u^{\prime}}{3}\right),
\end{aligned}
$$

so the natural asymmetry is

$$
\Delta r_{\text {nat }}=\left(u^{\prime}-u\right) r_{h} \approx \frac{2}{3}\left(\frac{m}{3 M}\right)^{2 / 3} r_{h} .
$$

Now introducing a fifth force, the force balance equations for stars not directly coupling to the fifth force become

$$
\begin{aligned}
& -\frac{G M}{\left(r_{h}-r_{t}\right)^{2}}+\frac{G m}{r_{t}^{2}}+\Omega^{2}\left(1+2 \beta^{2}\right)\left(\frac{M r_{h}}{M+m}-r_{t}\right)=0, \\
& -\frac{G M}{\left(r_{h}+r_{t}\right)^{2}}-\frac{G m}{r_{t}^{2}}+\Omega^{2}\left(1+2 \beta^{2}\right)\left(\frac{M r_{h}}{M+m}+r_{t}\right)=0 .
\end{aligned}
$$

Proceeding as before

$$
\begin{aligned}
& u \approx\left(\frac{m}{3 M}\right)^{1 / 3} \frac{1}{\left(1+2 \beta^{2}\right)^{1 / 3}}\left(1-\frac{u}{3}+\frac{2 \beta^{2}}{3} \frac{M}{m} u^{2}\right), \\
& u^{\prime} \approx\left(\frac{m}{3 M}\right)^{1 / 3} \frac{1}{\left(1+2 \beta^{2}\right)^{1 / 3}}\left(1+\frac{u^{\prime}}{3}-\frac{2 \beta^{2}}{3} \frac{M}{m} u^{\prime 2}\right) .
\end{aligned}
$$

The last term on the right-hand side produces an asymmetry with opposite sign to the natural asymmetry. Note that as $u \propto(m / M)^{1 / 3}$, the $M / m$ factor makes this term actually the largest. The condition for the asymmetry due to the fifth force to overwhelm the Newtonian one is then just

$$
2 \beta^{2} \gtrsim 3^{1 / 3}\left(\frac{m}{M}\right)^{2 / 3}
$$

where only leading terms are kept. This result can be compared with Eq. (29) of Ref. [13]. Although the scaling is the same, the numerical factor is different (remember on comparing results that $2 \beta^{2}$ in our paper corresponds to $\beta^{2} f_{R} f_{\text {sat }}$ in theirs). In fact, the changes are very much to the advantage of the fifth force, as smaller values of $\beta$ now give detectable asymmetries.

The two most massive of the Milky Way (MW) dwarf spheroidals are Sagittarius with dark matter mass $2.8 \times$ $10^{8} M_{\odot}$ and Fornax at $1.3 \times 10^{8} M_{\odot}$ [39]. These will allow values of $\beta^{2} \gtrsim 2 \times 10^{-3}$ to be probed. For the smallest dwarf spheroidals such as Segue 1 with a mass of 
$6 \times 10^{5} M_{\odot}$, then values of $\beta^{2} \gtrsim 2 \times 10^{-4}$ are in principle accessible. It should be noted that Segue 1 is an ambiguous object, and it is not entirely clear if it is a dark-matterdominated dwarf or a globular cluster [40].

\section{General case}

The circular restricted three-body problem is somewhat unrealistic, as the Galaxy's matter distribution is extended. In particular, there is a significant difference in the enclosed host mass within the inner and outer Lagrange points and this plays a role in the strength of the asymmetry. We now proceed to give a mathematical analysis of the general case.

The satellite is now moving on a orbit with instantaneous angular frequency $\boldsymbol{\Omega}$. We work in a (noninertial) reference frame rotating at $\boldsymbol{\Omega}$ with origin at the center of the satellite. A star at location $\mathbf{r}_{s}$ now feels the following forces: (i) a gravitational attraction by the satellite, (ii) a gravitational attraction by the host galaxy; (iii) an inertial force because the satellite is falling into the host and so the reference frame is not inertial; and (iv) the Euler, Coriolis and centrifugal forces because the reference frame is rotating. Note that (iii) was not necessary in our earlier treatment of the circular restricted three-body problem because there we chose an inertial frame tied to the center of mass.

The equation of motion for a star or dark matter particle is

$$
\begin{aligned}
\ddot{\mathbf{r}}_{s}= & -G m\left(r_{\mathrm{s}}\right) \frac{\mathbf{r}_{s}}{r_{s}{ }^{3}}-G M\left(\left|\mathbf{r}_{s}-\mathbf{r}_{h}\right|\right) \frac{\left(\mathbf{r}_{s}-\mathbf{r}_{h}\right)}{\left|\mathbf{r}_{s}-\mathbf{r}_{h}\right|^{3}} \\
& -G M\left(r_{h}\right) \frac{\mathbf{r}_{h}}{r_{h}{ }^{3}}-\dot{\boldsymbol{\Omega}} \times \mathbf{r}_{s}-2 \boldsymbol{\Omega} \times \dot{\mathbf{r}}_{s} \\
& -\boldsymbol{\Omega} \times\left(\boldsymbol{\Omega} \times \mathbf{r}_{s}\right) .
\end{aligned}
$$

Save for the assumption that the matter distributions in the satellite $m\left(r_{s}\right)$ and the host $M\left(r_{h}\right)$ are spherically symmetric, this expression is general.

We now assume that the star or dark matter particle is following a circular orbit around the satellite with orbital frequency $\boldsymbol{\Omega}_{s}$ and that $r_{s} / r_{h} \ll 1$. By careful Taylor expansion, we obtain

$$
\begin{aligned}
\ddot{\mathbf{r}}_{s}= & -G m\left(r_{s}\right) \frac{\mathbf{r}_{s}}{r_{s}{ }^{3}}+G M\left(r_{h}\right) \frac{(3-n)\left(\mathbf{r}_{s} \cdot \mathbf{r}_{h}\right) \mathbf{r}_{\mathrm{h}}}{\left|r_{\mathrm{h}}\right|^{5}} \\
& -G M\left(r_{h}\right) \frac{\mathbf{r}_{s}}{r_{h}{ }^{3}}-\dot{\boldsymbol{\Omega}} \times \mathbf{r}_{s}-2 \boldsymbol{\Omega} \times\left(\boldsymbol{\Omega}_{s} \times \mathbf{r}_{s}\right) \\
& +\boldsymbol{\Omega} \times\left(\boldsymbol{\Omega} \times \mathbf{r}_{s}\right),
\end{aligned}
$$

where $n\left(r_{h}\right)$ is the logarithmic gradient of $M\left(r_{h}\right)$.

To calculate the tidal radius, we now specialize to the case of a particle whose orbit lies in the same plane as the satellite's orbit. The satellite's circular frequency is $\Omega^{2}=G M\left(r_{h}\right) / r_{h}{ }^{3}$. The tidal radius is defined as the distance from the center of the satellite at which there is no net acceleration, i.e., the forces on the particle toward the host and the satellite balance. This gives the tidal radius as

$$
r_{t}=\frac{1}{\left(1-n+2 \Omega_{s} / \Omega\right)^{1 / 3}}\left(\frac{m\left(r_{s}\right)}{M\left(r_{h}\right)}\right)^{1 / 3} r_{h} .
$$

When satellite and host are point masses, then $n=0$ and $\Omega=\Omega_{s}$, and we recover the result previously found in Eq. (6).

We now define $u=r_{t} / r_{h}$ and $u^{\prime}=r_{t}^{\prime} / r_{h}$ for the inner and outer Lagrange points, respectively, and obtain

$$
\begin{gathered}
u^{3}=\frac{m\left(r_{s}\right)}{M\left(r_{h}\right)} \frac{(1-u)^{2-n} u}{1-(1-u)^{2-n}+\alpha(1-u)^{2-n} u}, \\
u^{\prime 3}=\frac{m\left(r_{s}\right)}{M\left(r_{h}\right)} \frac{\left(1+u^{\prime}\right)^{2-n} u^{\prime}}{\left(1+u^{\prime}\right)^{2-n}-1+\alpha\left(1+u^{\prime}\right)^{2-n} u^{\prime}},
\end{gathered}
$$

where $\alpha \equiv 2 \Omega_{s} / \Omega-1$. We now solve for the difference in the positions of the Lagrange points with respect to the satellite center $u^{\prime}-u$. This is the natural stream asymmetry

$\Delta r_{\text {nat }} \approx\left(u^{\prime}-u\right) r_{h}=\left(\frac{m\left(r_{s}\right)}{M\left(r_{h}\right)}\right)^{2 / 3} \frac{(2-n)(3-n) r_{h}}{3\left(1-n+2 \Omega_{s} / \Omega\right)^{5 / 3}}$.

In the restricted three-body problem, $n=0$ and $\Omega=\Omega_{s}$, so we recover our previous result in Eq. (16).

We wish to compare this asymmetry to the asymmetry produced by adding the modified gravity acceleration of the satellite to the equation of motion. Now specializing to the case $\Omega=\Omega_{s}$ to reduce complexity, we find the asymmetry due to the fifth force is

$$
\Delta r_{5} \approx-\frac{4}{3(3-n)} \beta^{2} r_{h} .
$$

So, the requirement that the dark matter asymmetry overwhelms the natural asymmetry is

$$
2 \beta^{2} \gtrsim \frac{(2-n)(3-n)}{2(3-n)^{2 / 3}}\left(\frac{m}{M}\right)^{2 / 3},
$$

which again reduces to Eq. (21) in the restricted three-body case, as it should. For galactic dynamics, a reasonable choice is $n=1$, which corresponds to a galaxy with a flat rotation curve, i.e., an isothermal sphere. Assuming the stars in the satellite satisfy $\Omega_{s}=\Omega$, then tidal streams in galaxies with flat rotation curves are much more sensitive probes of the dark matter asymmetry. As we move from $n=0$ (the point mass case) to $n=1$ (the isothermal sphere), we gain an additional factor of $\approx 2.3$ in sensitivity. The changes are again in our favor. The asymmetries in tidal streams are therefore an even more delicate probe of the fifth force than suggested by the analysis in Ref. [13]. 


\section{MILKY WAY AND SATELLITE MODELS}

In our simulations, we follow the evolution of a large number of tracer particles, stripped from a satellite galaxy and forming tidal tails. The test particles are accelerated by the gravity field of both the Milky Way and the satellite, together with any fifth force contributions. We begin by describing our models for the Milky Way and satellite.

\section{A. Milky Way model}

For the Milky Way, we adopt the axisymmetric mass model of Ref. [41], which is designed to fit a number of recent kinematic constraints on the Milky Way matter distribution. The model comprises six distinct components: a central bulge, a dark matter halo, and four disks (thin and thick stellar disks and atomic and molecular gas disks). The functional form of the density distribution of each of these components is given as follows. The various undefined symbols are parameters of the model, for all of which we adopt the values of Ref. [41]. We reproduce these values in Table I.

For the DM halo, the model employs a Navarro-FrenkWhite profile [42]:

$$
\rho(r)=\frac{\rho_{0, h}}{\left(\frac{r}{r_{0, h}}\right)\left(1+\frac{r}{r_{0, h}}\right)^{2}} .
$$

We have experimented with nonspherical oblate and prolate halo profiles, as well as steeper inner slopes (cf. [43]), and found no significant impact on our results. However, it would be interesting in future to investigate the impact of a truly triaxial dark matter halo.

Meanwhile, the bulge is represented by an axisymmetrized version of the model of Bissantz and Gerhard [44]:

$$
\rho(R, z)=\frac{\rho_{0, b}}{\left(1+\frac{r^{\prime}}{r_{0, b}}\right)^{1.8}} e^{-\left(r^{\prime} / r_{\text {cut }}\right)^{2}}
$$

where $r^{\prime} \equiv \sqrt{R^{2}+4 z^{2}}$.

The two stellar disks are represented by a simple exponential disk with an exponential vertical profile:

$$
\rho(R, z)=\frac{\Sigma_{0}}{2 z_{0}} e^{-R / R_{0}} e^{-|z| / z_{0}}
$$

while the two gas disks are given by an exponential disk model with a central hole and a "sech-squared" vertical profile:

$$
\rho(R, z)=\frac{\Sigma_{0}}{4 z_{0}} e^{-\left(R_{h} / R+R / R_{0}\right)} \operatorname{sech}^{2}\left(\frac{z}{2 z_{0}}\right)
$$

With this axisymmetric mass model in hand, the gravitational potential is then calculated with a Poisson solver [45] utilizing a spherical harmonic technique similar to that described by Dehnen and Binney [46]. The solver calculates the potential and its gradients on a spherical grid; for

TABLE I. Milky Way model parameters from Ref. [41]. The first three columns, respectively, give the symbol representing a given parameter, the number of the equation in which it appears, and a physical description. The final column lists the parameter values; in most cases these are best-fitting values inferred by Ref. [41], but some were instead fixed $a$ priori, such as the various disk scale heights. Further details can be found in that article.

\begin{tabular}{lllcc}
\hline \hline Symbol & Equation & \multicolumn{1}{c}{ Parameter } & \multicolumn{2}{c}{ Value } \\
\hline$\rho_{0, h}$ & $(30)$ & Halo scale density & 0.00853702 & $M_{\odot} / \mathrm{pc}^{3}$ \\
$r_{0, h}$ & $(30)$ & Halo scale radius & 19.5725 & $\mathrm{kpc}$ \\
$\rho_{0, b}$ & $(31)$ & Bulge scale density & 98.351 & $M_{\odot} / \mathrm{pc}^{3}$ \\
$r_{0, b}$ & $(31)$ & Bulge scale radius & 0.075 & $\mathrm{kpc}$ \\
$r_{\text {cut }}$ & $(31)$ & Bulge cutoff radius & 2.1 & $\mathrm{kpc}$ \\
$\Sigma_{0}^{\text {thin }}$ & $(32)$ & Thin disk normalization & 895.679 & $M_{\odot} / \mathrm{pc}^{2}$ \\
$z_{0}^{\text {thin }}$ & $(32)$ & Thin disk scale height & 300 & $\mathrm{pc}$ \\
$R_{0}^{\text {thin }}$ & $(32)$ & Thin disk scale radius & 2.49955 & $\mathrm{kpc}$ \\
$\Sigma_{0}^{\text {thick }}$ & $(32)$ & Thick disk normalization & 183.444 & $M_{\odot} / \mathrm{pc}^{2}$ \\
$z_{0}^{\text {thick }}$ & $(32)$ & Thick disk scale height & 900 & $\mathrm{pc}$ \\
$R_{0}^{\text {thick }}$ & $(32)$ & Thick disk scale radius & 3.02134 & $\mathrm{kpc}$ \\
$\Sigma_{0}^{\mathrm{HI}}$ & $(33)$ & HI disk normalization & 53.1319 & $M_{\odot} / \mathrm{pc}^{2}$ \\
$z_{0}^{\mathrm{HI}}$ & $(33)$ & HI disk scale height & 85 & $\mathrm{pc}$ \\
$R_{0}^{\mathrm{HI}}$ & $(33)$ & HI disk scale radius & 7 & $\mathrm{kpc}$ \\
$R_{h}^{\mathrm{HI}}$ & $(33)$ & HI disk hole radius & 4 & $\mathrm{kpc}$ \\
$\Sigma_{0}^{\mathrm{H}_{2}}$ & $(33)$ & $\mathrm{H}_{2}$ disk normalization & 2179.95 & $M_{\odot} / \mathrm{pc}^{2}$ \\
$z_{0}^{\mathrm{H}_{2}}$ & $(33)$ & $\mathrm{H}_{2}$ disk scale height & 45 & $\mathrm{pc}$ \\
$R_{0}^{\mathrm{H}_{2}}$ & $(33)$ & $\mathrm{H}_{2}$ disk scale radius & 1.5 & $\mathrm{kpc}$ \\
$R_{h}^{\mathrm{H}_{2}}$ & $(33)$ & $\mathrm{H}_{2}$ disk hole radius & 12 & $\mathrm{kpc}$ \\
\hline \hline
\end{tabular}


our simulations we use a grid with 2000 log-spaced radial cells between $10^{-4}$ and $10^{4} \mathrm{kpc}$, and 2500 (polar) angular cells, and truncate the spherical harmonic expansion at multipole $l=80$. These settings were found to yield converged solutions for the Milky Way potential corresponding to the mass model described above.

The gravitational acceleration on a test particle (neglecting any fifth forces for the moment) due to the Milky Way is then calculated by interpolating the potential gradient at the position of the particle, employing a cubic spline.

\section{B. Satellite model}

We model the satellite with a truncated Hernquist sphere with the density cut off at a radius $r_{\mathrm{t}}$. The reason for this sharp truncation will become clear in the discussion of the fifth force in Sec. III C. Defining a reduced radius $x \equiv r / a_{\mathrm{S}}$ (thus $x_{t} \equiv r_{t} / a_{\mathrm{S}}$ ), where $a_{\mathrm{S}}$ is the scale radius of the profile, the density-potential pair is given by

$$
\begin{aligned}
& \Phi(x)= \begin{cases}-\frac{G m}{r_{t}}\left[1+\frac{\left(1+x_{t}\right)^{2}}{x_{t}}\left(\frac{1}{1+x}-\frac{1}{1+x_{t}}\right)\right], & x \leq x_{t}, \\
-\frac{G m}{r}, & x>x_{t},\end{cases} \\
& \rho(x)= \begin{cases}\frac{A}{x(1+x)^{3}}, & x \leq x_{t}, \\
0, & x>x_{\mathrm{t}} .\end{cases}
\end{aligned}
$$

The density normalization $A$ is related to the total satellite mass $m$ by

$$
A=\frac{\left(1+x_{t}\right)^{2}}{x_{t}^{2}} \frac{m}{2 \pi a^{3}} .
$$

The mass enclosed within a reduced radius $x$ is then

$$
m(x)= \begin{cases}m \frac{x^{2}\left(1+x_{t}\right)^{2}}{x_{t}^{2}(1+x)^{2}}, & \text { if } x \leq x_{t}, \\ m, & \text { otherwise. }\end{cases}
$$

For all satellites, we adopt truncation radii of $r_{\mathrm{t}}=10 a_{\mathrm{S}}$ or, equivalently, $x_{t}=10$.

The acceleration on any given test particle due to the satellite can then be calculated from the above relations. For self-consistency, the initial phase-space distribution of the tracer particles is that of a truncated Hernquist profile (see Sec. IVA for further details). Of course, this selfconsistency is lost as the simulation advances in time, as many of the tracer particles are tidally removed by the Milky Way, but our assumed satellite potential remains unchanged in mass and shape. However, we will show in Sec. V that this assumption of an unchanging satellite potential is largely harmless.

\section{Fifth forces}

In addition to gravity, the satellite and the tracer particles also experience accelerations due to the fifth force. The satellite feels a fifth force sourced by the Milky Way, while the tracer particles also feel a fifth force sourced by the satellite. We assume spherical fifth force profiles in both cases. For the satellite, this is consistent with its gravitational potential, although the sphericity of the satellite may be distorted by its tidal disruption. For the Milky Way, the spherical symmetry is inconsistent with the presence of the disk. The scalar field profiles of disk galaxies have correspondingly discoid shapes [28]. However, the scalar field profile is roughly spherical when $r_{\text {scr,Mw }}$ is much larger than the disk scale radius of $6.5 \mathrm{kpc}$. In particular, using the $f(R)$ scalar field solver described in Sec. VID, we find that fifth force profiles in the Milky Way (assuming a spherical dark matter halo) only become appreciably aspherical for $\log _{10}\left|f_{R 0}\right| \gtrsim-6.2$, and so the spherical approximation is robust in the parameter regimes we mostly focus on in this article.

Equation (2) can be rewritten to give the expression for the modified gravity acceleration due to the satellite on tracer particle $i$, situated at position $\boldsymbol{x}$ :

$$
\boldsymbol{a}_{5, \text { sat }}^{i}(\boldsymbol{x})=2 \beta^{2} Q_{i} Q_{\mathrm{sat}}(r) \boldsymbol{a}_{\mathrm{N}, \mathrm{sat}}(\boldsymbol{x}),
$$

where $\beta$ is the coupling strength of the fifth force (an input parameter of our simulations), $\boldsymbol{a}_{\mathrm{N} \text {,sat }}$ is the Newtonian acceleration due to the satellite, and $Q_{i}$ and $Q_{\text {sat }}(r)$ are the scalar charges of particle $i$ and the satellite, respectively. The latter is given by

$$
Q_{\text {sat }}(r)= \begin{cases}1-\frac{m\left(r_{\text {scr,sat }}\right)}{m}, & \text { if } r \geq r_{t}, \\ 1-\frac{m\left(r_{\text {scr,sat }}\right)}{m(r)}, & \text { if } r_{t}>r \geq r_{\text {scr,sat }}, \\ 0, & \text { otherwise. }\end{cases}
$$

Here, $m(r)$ is the satellite mass enclosed by radius $r$, and $r_{\text {scr,sat }}$ is its screening radius. $Q_{i}$, meanwhile, differs between the particle types. As we assume the stars are fully screened against the fifth force, $Q_{i}=0$ for the star tracer particles. On the other hand, we take $Q_{i}=1$ for the dark matter tracer particles, which we assume to be a diffuse, unscreened component.

Similarly, the modified gravity acceleration due to the Milky Way on particle $i$ (which can now also represent the satellite) at $\boldsymbol{x}$ is given by

$$
\boldsymbol{a}_{5, \mathrm{MW}}^{i}(\boldsymbol{x})=2 \beta^{2} Q_{i} Q_{\mathrm{MW}}(r) \boldsymbol{a}_{\mathrm{N}, \mathrm{MW}}(\boldsymbol{x}),
$$

where the symbols have analogous meanings to those above. The scalar charge of the Milky Way is given by 


$$
Q_{\mathrm{MW}}(r)=\left\{\begin{array}{lc}
1-\frac{M\left(r_{\mathrm{scr}, \mathrm{MW}}\right)}{M(r)}, & \text { if } r \geq r_{\mathrm{scr}, \mathrm{MW}} \\
0, & \text { otherwise }
\end{array}\right.
$$

If particle $i$ represents the satellite, then we take the limiting value of the satellite scalar charge $Q_{i}=Q_{\text {sat }}\left(r=r_{t}\right)$. This is valid as long as the Milky Way center does not fall within the truncation radius of the satellite center, which does not happen in any of our simulations.

The formalism given in this subsection demonstrates the utility of truncating the mass profile of the satellite. By so doing, we have made it straightforward to model the satellite as being fully screened $\left(r_{\text {scr,sat }}=r_{t}\right)$, fully unscreened $\left(r_{\text {scr,sat }}=0\right)$, or partially screened $\left(0<r_{\text {scr,sat }}<r_{t}\right)$.

It is worth remarking that we have used the superposition principle to compute the joint fifth force of Milky Way and satellite on the tracer particles. Strictly speaking, the superposition is not valid in highly nonlinear theories of gravity like chameleon gravity. In particular, environmental screening can affect the screening radii of objects. Linearity is, however, restored once the screening radii are fixed (as we do by hand), so that from that point on we can apply the superposition principle for computing the joint fifth force.

\section{METHODS}

Approximate methods for quickly generating realistic streams by stripping stars at the tidal radius of a progenitor are now well established [47-49]. The methods work as restricted $N$-body simulations, in which we follow the orbital evolution of a large number of massless tracer particles. The stream particles are integrated in a fixed Galactic potential, together with the potential of the moving satellite. This method robustly reproduces the morphology of streams, in particular the locations of the apocenters of the leading and trailing branches, yet provides 2-3 orders of magnitude speed-up compared to conventional $N$-body experiments [49]. The main extension of our code here is that it incorporates an optional fifth force due to the chameleon field.

All of our code is made publicly available as the PYTHON3 package smoggy (streams under modified gravity) [50]. Animations of the simulations depicted in Figs. 4, 6,10 , and 14 are given as Supplemental Material accompanying this article [51].

\section{A. Tracer particles}

To generate the initial phase space distribution of $N$ tracer particles, we use a Markov chain Monte Carlo technique to generate $2 \mathrm{~N}$ samples from possible equilibrium distribution functions (DFs) for the Hernquist model. The choice of equilibrium includes the isotropic DF [52]

$$
\begin{aligned}
f(\tilde{E})= & \frac{1}{\sqrt{2}(2 \pi)^{3}\left(G M^{\prime} a_{\mathrm{S}}\right)^{3 / 2}} \frac{\sqrt{\tilde{E}}}{(1-\tilde{E})^{2}} \\
& \times\left[(1-2 \tilde{E})\left(8 \tilde{E}^{2}-8 \tilde{E}-3\right)+\frac{3 \sin ^{-1} \sqrt{\tilde{E}}}{\sqrt{\tilde{E}(1-\tilde{E})}}\right]
\end{aligned}
$$

and the radially anisotropic DF [53]

$$
f(\tilde{E})=\frac{3}{4 \pi^{3} a_{\mathrm{S}}} \frac{\tilde{E}}{G L} .
$$

Here, $E$ is the specific (binding) energy of a particle, $a_{\mathrm{S}}$ is the scale radius, and $M^{\prime}=\left(1+x_{t}\right)^{2} m / x_{t}^{2}$ is the untruncated mass of the satellite, while $\tilde{E}=E a_{\mathrm{S}} / G M^{\prime}$ is the dimensionless binding energy. The DFs differ in the anisotropy of the velocity distributions. In fact, our simulations show similar results for stream generation, irrespective of the anisotropy, so the choice of equilibrium is not so important.

Given these $2 N$ samples, we integrate the orbits of the particles in the satellite potential (i.e., neglecting fifth forces and the Milky Way) for $10^{17} \mathrm{~s}(\approx 3 \mathrm{Gyr})$. At the end of this relaxation phase, we randomly downsample $N$ of these particles, excluding any particles for which the orbit ever strayed beyond the truncation radius. This gives a suitable equilibrium distribution of positions and velocities for the test particles in our simulations.

All of our simulations incorporate $10000 \mathrm{DM}$ particles and 10000 star particles. This equality does not encode any assumptions about the underlying stellar or DM mass fraction of the satellite; the particles are merely massless samples of the distribution, and the satellite is assumed to be dark matter dominated.

This procedure has omitted the fifth force altogether. This is appropriate for the star particles which, by assumption, do not experience the fifth force. For the dark matter however, it is less self-consistent. We have experimented with including a fifth force, both in the distribution function and in the relaxation phase described in the previous paragraph. This leads overall to $\sim 10 \%$ increases in the number of dark matter particles being stripped from the progenitor during the main simulation, but no appreciable morphological change to the dark matter streams.

Note also that this procedure makes the unrealistic assumption that the stars and dark matter have the same spatial distribution. However, we have experimented with drawing the stars from more compact initial distributions than the dark matter and found no appreciable difference in our results.

\section{B. Orbit integration}

To calculate the trajectories of the various particles, we use a second-order leapfrog integrator. Under such a scheme, the velocities $\boldsymbol{v}$ and positions $\boldsymbol{x}$ of the particles are updated at each time step $i$ via 
TABLE II. Parameters for each of the four progenitors. Here, $\boldsymbol{x}_{0}$ and $\boldsymbol{v}_{0}$ give the present position and velocity, respectively (note that we run the simulations backward and then forward again, so that the satellites end at $\boldsymbol{x}_{0}$ and $\left.v_{0}\right), a$ and $m$ are the Hernquist scale radius and total mass of the satellite, respectively, and $t_{\max }$ is the total time over which each simulation is run; the farther orbits require more time to undergo an appreciable number of orbital periods. Note that $10^{17} \mathrm{~s}$ is $\sim 3$ Gyr. The parameters for satellite A resemble the Pal-5 stream, B the Sagittarius stream, $\mathrm{C}$ the orphan stream, and D a hypothetical stream at a large distance.

\begin{tabular}{lccccc}
\hline \hline ID & $\boldsymbol{x}_{0}(\mathrm{kpc})$ & $\boldsymbol{v}_{0}(\mathrm{~km} / \mathrm{s})$ & $a(\mathrm{kpc})$ & $m\left(10^{8} M_{\odot}\right)$ & $t_{\max }\left(10^{17} \mathrm{~s}\right)$ \\
\hline $\mathrm{A}$ & $(7.7,0.2,16.4)$ & $(-44,-117,-16)$ & 0.01 & 0.0003 & 1 \\
$\mathrm{~B}$ & $(19.0,2.7,-6.9)$ & $(230,-35,195)$ & 0.5 & 5 & 1 \\
$\mathrm{C}$ & $(90,0,0)$ & $(0,0,80)$ & 0.5 & 2.5 & 1.5 \\
$\mathrm{D}$ & $(150,0,0)$ & $(0,0,100)$ & 1 & 5 & 2.5 \\
\hline \hline
\end{tabular}

$$
\begin{aligned}
\boldsymbol{v}_{i+1 / 2} & =\boldsymbol{v}_{i-1 / 2}+\boldsymbol{a}\left(\boldsymbol{x}_{i}\right) \Delta t, \\
\boldsymbol{x}_{i+1} & =\boldsymbol{x}_{i}+\boldsymbol{v}_{i+1 / 2} \Delta t,
\end{aligned}
$$

where $\Delta t$ represents the time step size and $\boldsymbol{a}(\boldsymbol{x})$ represents the accelerations calculated using the expressions given in Secs. III A-III C. At the start of the simulation (i.e., time step $i=0$ ), the "desynchronized" velocities $\boldsymbol{v}_{-1 / 2}$ are obtained using

$$
v_{-1 / 2}=v_{0}-\frac{1}{2} a\left(x_{0}\right) \Delta t
$$

From here, Eq. (43) can be used repeatedly to advance the system in time.

Our method for choosing the time step size $\Delta t$ is as follows. We calculate the total energies of all particles at the start and end of the relaxation phase described in Sec. IV A, in which the orbits are integrated in the satellite potential for $10^{17} \mathrm{~s}$. We repeat the relaxation phase, iteratively reducing the time step size, until the energies of all particles are conserved to within $2 \%$. Through experimentation, we found that energy conservation is a good proxy for numerical convergence and this $2 \%$ criterion gives accurate, converged results. With this criterion, we find that angular momentum is conserved to an even greater precision, with a maximum fractional deviation of $\sim 10^{-4}$. The final time step size chosen by this process is then used again for the main simulation. In practice, we find $\Delta t \sim \mathcal{O}\left(10^{11}\right)$ s typically.

\section{Simulations}

We simulate the generation of streams from four progenitors. Satellite A is inspired by the Palomar 5 stream [54], B the Sagittarius stream [55], C the orphan stream [8], and $\mathrm{D}$ is a hypothetical stream at a large Galactocentric distance, of the kind that is likely to be found in the later Gaia data releases. The parameters for these four progenitors are given in Table II.

Figure 2 shows the evolution of the orbits over $\sim 3 \mathrm{Gyr}$ for each of the four satellites, under standard gravity. Also shown are lines indicating the disk-plane Milky Way screening radii for a range of values of $f_{R 0}$. These calculations were performed using the scalar field solver within the $f(R) N$-body code MG-Gadget [56] for the Milky Way model described in Sec. III A. We demonstrate later that significant stream asymmetries develop when the orbit is mostly outside the Milky Way screening radius, so these lines give a preview of the modified gravity constraints achievable.

For each satellite, we explore a variety of modified gravity scenarios by varying three input parameters: the coupling strength $\beta$, the satellite screening radius $r_{\text {scr,sat }}$,

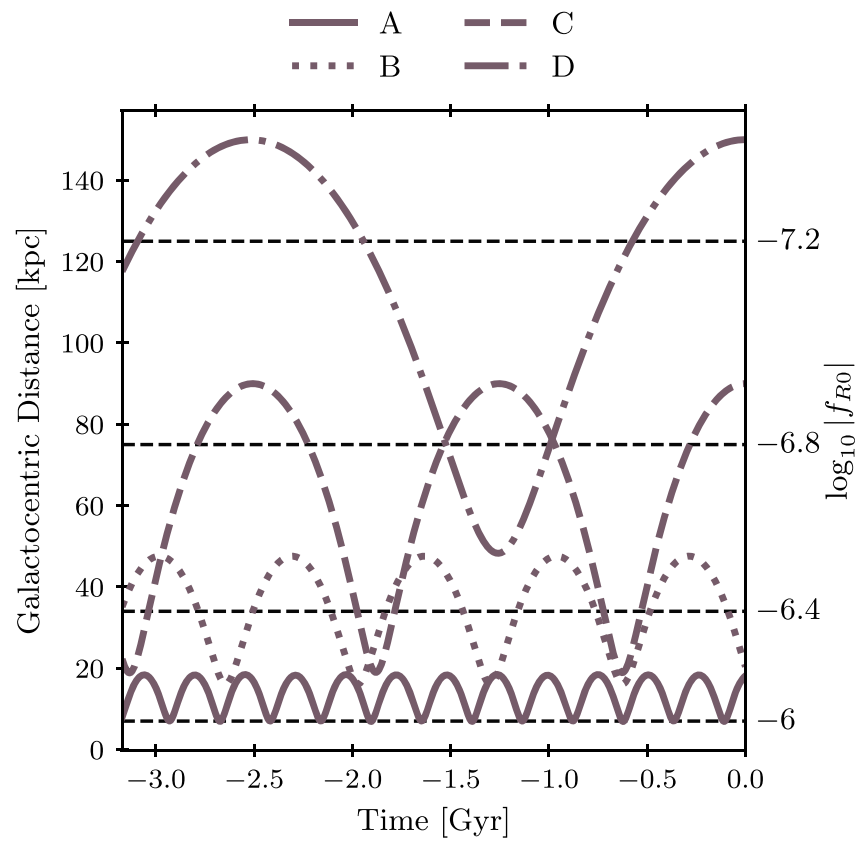

FIG. 2. For the four satellites described in Table II, we show the orbital evolution over $10^{17} \mathrm{~s}(\sim 3 \mathrm{Gyr})$ under standard gravity. The horizontal dashed lines indicate the Milky Way screening radii under Hu-Sawicki $f(R)$ gravity for various different values of the theory parameter $f_{R 0}$ (the values of $\log _{10}\left|f_{R 0}\right|$ are shown at the right-hand side of the panel) (see Sec. IV C for details about the calculation of these screening radii). This figure illustrates the range of distances probed by tidal streams and gives an idea of the possible constraints achievable for chameleon gravity theories. 
and the Milky Way screening radius $r_{\text {scr,Mw. First, we }}$ consider four coupling strengths: $\beta=\{0.1,0.2,0.3,0.4\}$. The strength of the fifth force relative to gravity is given by $2 \beta^{2}$, so this corresponds to the range from $2 \%$ to $32 \%$. The most extreme case can therefore be used as an approximate analog for $f(R)$ gravity, where the strength of the fifth force is $1 / 3$ that of gravity.

For the satellite screening radius, we explore a range of regimes, from fully screened to fully unscreened, and encompassing a variety of partially screened regimes in between. Using the upper case of Eq. (38), we recast the screening radius $r_{\text {scr,sat }}$ as the scalar charge $Q_{\text {sat }}$ and consider a range of values of $Q_{\text {sat }}$ from 0 to 1 in steps of 0.1 . We recall that $Q_{\text {sat }}=0$ corresponds to the fully screened case, so here $r_{\text {scr,sat }}=10 a$, where $a$ is the Hernquist scale radius of the satellite in question. $Q_{\text {sat }}=1$ is the fully unscreened case, so $r_{\text {scr,sat }}=0$.

Finally, we consider a range of values for the Milky Way screening radius $r_{\text {scr,Mw. }}$. As the orbital distances of each satellite are different, it is useful to select a different range of values for $r_{\text {scr,Mw }}$ for each satellite. For each satellite, we define a maximum screening radius $r_{\text {scr,max }}$, approximately equal to the apocentric distance of the orbit under standard gravity. These values are $r_{\text {scr,max }}=20,50,90$, and $150 \mathrm{kpc}$ for satellites A, B, C, and D, respectively. Then, we choose a range of 11 values such that $r_{\mathrm{scr}, \mathrm{MW}} / r_{\mathrm{scr}, \max }$ runs from 0 to 1 in steps of 0.1 .

Altogether, we run 485 simulations for each satellite: $4 \times 11 \times 11=484$ modified gravity simulations plus one standard gravity $(\beta=0)$ simulation.

\section{Assumptions}

The previous subsections have given details about the various parts of our code, but for clarity we provide a list of all of our simplifying assumptions.

(1) We neglect self-gravity between the tracer particles, both before and after they are stripped from the satellite, as is typical in Lagrange stripping codes $[11,49]$.

(2) We assume the gravitational attraction on the tracer particles due to the satellite can be approximated as that due to a (truncated) Hernquist sphere, whose orbit is only governed by the Milky Way potential. This assumption has been verified against full $N$-body simulations of stream formation by others [47-49].

(3) We assume the depth and radial extent of the satellite potential well does not change over time. While this assumption could be relaxed in the standard gravity case, it is a greatly helpful one in the chameleon case. Thus, to allow a fair comparison between results in the two cases, we make the assumption universally.

(4) We assume a static, axisymmetric model for the Milky Way potential, composed of a disk, bulge, and halo. Dynamical friction is therefore not modeled, though the effect is negligible at these low mass ratios [57]. We neglect any effects due to the Large Magellanic Cloud or other Milky Way satellites (cf. [8]).

(5) While we typically sample equal numbers of stellar and dark matter particles, we assume the mass profiles of our satellites to be dark matter dominated. So, the satellites feel the full fifth force in the absence of screening.

(6) The initial density profile and kinematics of the stellar and dark matter particles in the satellites are assumed to be the same. This simplifies the fifth force calculation and allows us to ensure any difference in the stellar and dark matter streams is due to the fifth force rather than initial conditions. As described in Sec. IV A, we have experimented with sampling the star particles from radially more compact distributions than the dark matter and found no significant difference in results.

Assumptions (1)-(6) apply equally in the standard gravity and modified gravity simulations. The following three assumptions, however, apply only in the simulations including a fifth force.

(7) We adopt spherical fifth force profiles around both the Milky Way and the satellite, despite the Milky Way potential being nonspherical. As discussed in Sec. III C, this is valid when the MW screening radius is larger than the Galactic disk, $\log _{10}\left|f_{R 0}\right| \lesssim-6.2$.

(8) Furthermore, we assume this spherical screening surface of the satellite remains fixed throughout the satellite's orbit. In reality, the radius would vary as the Galactocentric distance of the satellite changes, due to environmental screening, and the shape of the screening surface (and surrounding fifth force profile) would likely become aspherical as the satellite approached the Milky Way's screening radius and nonlinear effects warp the screening surface.

(9) The Compton wavelength of the scalar field is assumed to be much larger than relevant length scales. In the context of Hu-Sawicki $f(R)$ gravity, the Compton wavelength is given by $\lambda_{\mathrm{C}} \approx$ $32 \sqrt{\left|f_{R 0}\right| / 10^{-4}}$ Mpc [58], so this assumption starts to break down at around $f_{R 0} \sim 10^{-8}$.

\section{CODE VALIDATION}

As validation, we compare the results of our code for disruption of the Sagittarius dwarf galaxy under standard Newtonian gravity with the results of Law and Majewski [55]. They simulate the formation of the stream using a full $N$-body disintegration of the satellite in a static Milky Way potential, so assumptions (1)-(3) in the list in Sec. IV are not made in their work. In other words, the gravitational 


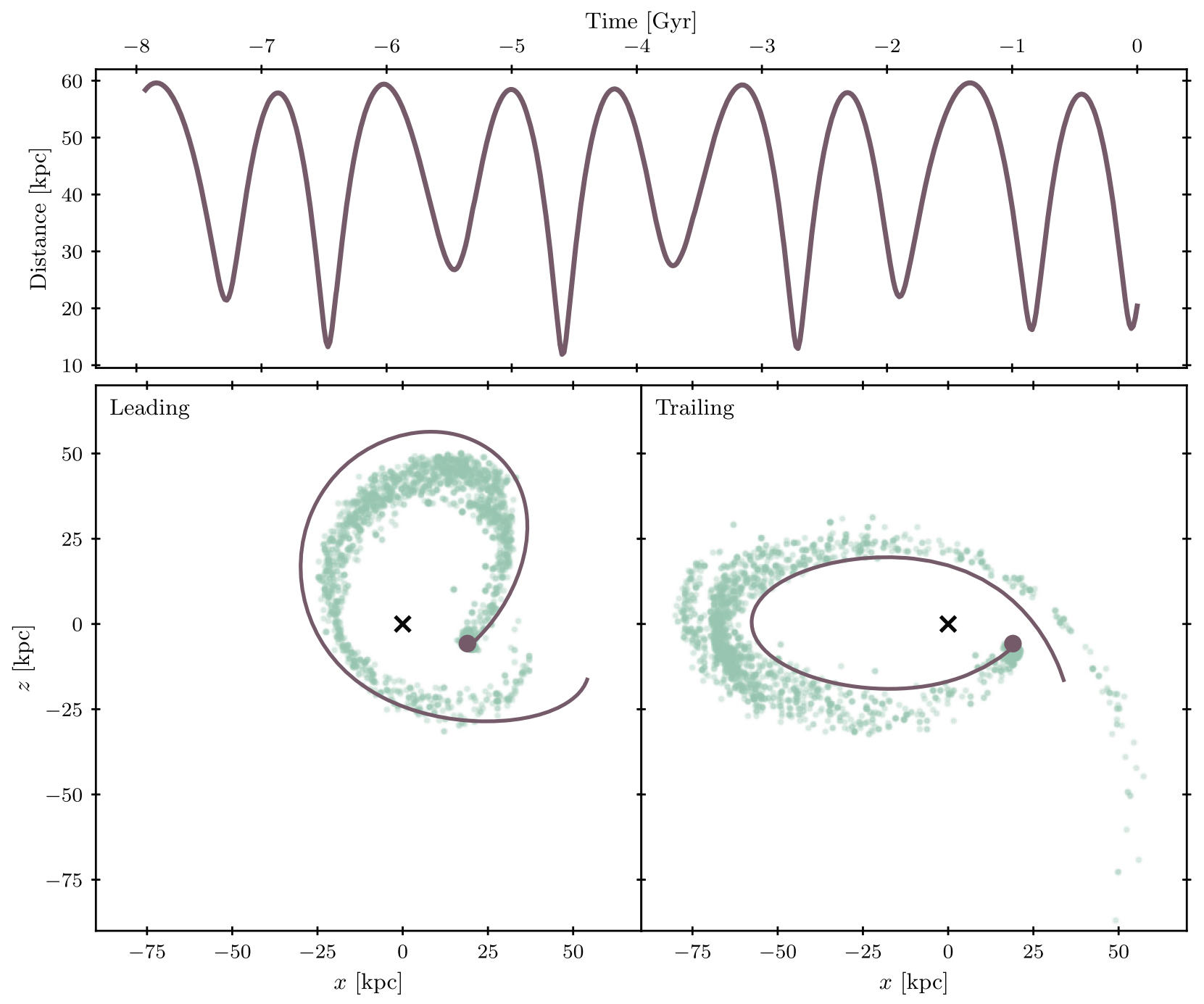

FIG. 3. Our reproduction of a simulation from Law and Majewski [55]. Top: Distance of the simulated Sagittarius dwarf from the Galactic center over 8 Gyr (to be compared to the results in Fig. 7 from Ref. [55]). Bottom left and right: First wrap of the leading and trailing streams, respectively (to be compared to the results in the two left-hand panels of Fig. 8 of Ref. [55]). The curve represents the orbital path of the satellite, culminating in the current position of the Sagittarius dwarf, represented by the filled circle. The green points are the positions of the simulation particles. The satellite orbit has been integrated over 3 Gyr up to the present day, so the morphology of the streams should resemble only the orange and magenta particles from the original figure. This successful reproduction of literature results serves as a test of our code and checks several of our simplifying assumptions.

attractions of the satellite and stream are there treated in a fully self-consistent manner.

To set up this test, we adopt the Milky Way potential of Ref. [55], i.e., a Hernquist bulge, a Miyamoto-Nagai disk, and a triaxial logarithmic dark matter halo. The parameters and initial conditions for the satellite are the same as those for satellite B, given in Table II.

As a first test, we integrate the orbit of the satellite in this potential backward for $2.5 \times 10^{17} \mathrm{~s}(\sim 8 \mathrm{Gyr})$. The distance of the satellite from the Galactic center as a function of time is shown in the upper panel of Fig. 3. This shows excellent agreement with Fig. 7 from Law and Majewski [55].

It is also desirable to check the morphology of the streams generated with our method. As a second test, we integrate the orbit of the satellite backward for $10^{17} \mathrm{~s}$ ( $\sim 3 \mathrm{Gyr})$ and then forward again with 16000 tracer particles. The resulting leading and trailing streams from this simulation are shown in the lower pair of panels in Fig. 3. The detailed morphologies of these streams closely resemble those of the streams depicted in Fig. 8 of Law and Majewski [55], considering only the orange and magenta particles in that figure (i.e., particles liberated within the last $3 \mathrm{Gyr}$ ).

Despite this reassuring agreement between the results from our simplified code and those from full $N$-body simulations, it is worth noting that several of the assumptions stated in Sec. IV D are not addressed by this test. In particular, this test does not validate the assumptions made 
in the treatment of the fifth force. However, the aim of the present work is to provide a qualitative understanding of the effects of chameleon gravity on stellar streams. Future work aiming to derive quantitative constraints from observational data will likely require either a relaxation or a more careful justification of some of those assumptions.

\section{RESULTS}

\section{A. Standard gravity}

Figure 4 shows the images from the standard gravity simulations for all four satellites listed in Table II. Each of the four quarters of the figure represents one of the satellites, as labeled in the top corner. The large subpanel in each quarter shows an image of the stream particles at the end of the simulation. As the stellar and dark matter particles are sampled from the same probability distribution initially (see assumption 6 in Sec. IV) and there is no EP violation by a fifth force in these standard gravity simulations, the stars and dark matter particles are indistinguishable and are thus not plotted separately in this figure. The three smaller subpanels in each quarter show the average velocity along the stream, velocity dispersion along the stream, and velocity dispersion perpendicular to the stream, all as a function of stream longitude and all calculated in bins of particles along the stream. The bins are created adaptively, such that each bin contains 25 particles, including only the particles which have been stripped from the progenitor. Within each bin, the unit vector giving the direction "along the stream" is taken as the (normed) average velocity vector of all particles in the bin. This figure illustrates the diversity of our simulated streams, with a variety of morphologies and Galactocentric distances represented.

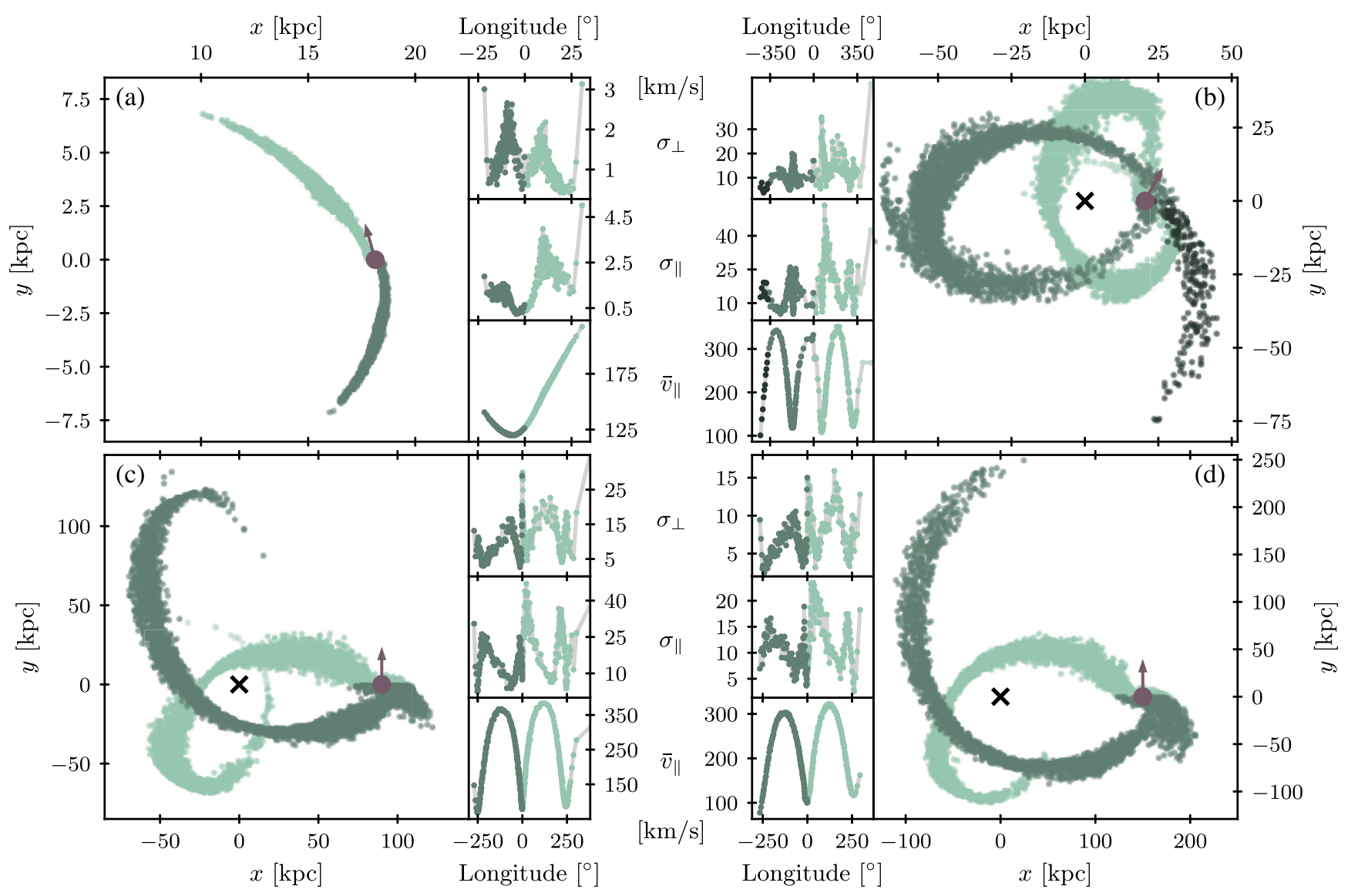

FIG. 4. The simulated streams under standard gravity. The four quarters represent our four satellites: A (upper left), B (upper right), C (lower left), and D (lower right). In each quarter, the largest subpanel shows an image of all stream particles in the orbital plane, at the end of the simulation. No distinction is made between star and dark matter particles. The colors differentiate leading and trailing streams, with the darker shade being the trailing stream. For satellite B, additional shades are used to distinguish multiple wraps. The black cross shows the position of the center of the Milky Way, while the filled circle shows the final position of the satellite, with an arrow indicating its instantaneous direction of travel. The side panels show three quantities calculated in bins of particles: average velocity along the stream, velocity dispersion along the stream, and velocity dispersion perpendicular to the stream. Here again, the colors differentiate leading and trailing streams. In every case, the orbital plane is defined such that the satellite is on the $x$ axis, moving in the positive $y$ direction. Animations of the four simulations depicted in this figure are included in Supplemental Material [51] accompanying this article. 


\section{B. Unscreened fifth force}

Turning to fifth forces, we first discuss results from an unscreened, EP-violating fifth force coupling only to dark matter $\left(r_{\text {scr,sat }}=r_{\text {scr,Mw }}=0\right)$. This is the case studied by Kesden and Kamionkowski $[13,14]$. This case also applies in screened modified gravity with a (formally) universal coupling if stars self-screen, but screening is not triggered otherwise. In our work, the strength of the fifth force relative to gravity is given by $2 \beta^{2}$, in keeping with the recent modified gravity literature, whereas Kesden and Kamionkowski used $\beta^{2}$. Thus, the simulation depicted in Fig. 6 for example $\left(\beta=0.2, F_{5} / F_{\mathrm{N}}=0.08\right)$, is most comparable to the " $\beta=0.3$ " $\left(F_{5} / F_{\mathrm{N}}=0.09\right)$ simulation in Refs. $[13,14]$.

Figure 5 shows the shape of satellite B's orbit for a variety of values of $\beta$. In the absence of screening, the introduction of a fifth force as in Eq. (1) is tantamount to an overall linear rescaling of the Milky Way mass or gravitational constant by a factor of $1+2 \beta^{2}$. As a consequence, the orbital period of the satellite is shorter and the apocentric distance smaller, as is apparent in Fig. 5.

Figure 6 shows the positions of the dark matter and star particles in the simulation with $r_{\text {scr,sat }}=r_{\text {scr, } \mathrm{MW}}=0$ and $\beta=0.2$ for satellite $\mathrm{C}$, at 11 equally spaced snapshots over time (recall that animations of selected simulations are available online). The most striking feature is the asymmetry of the stellar stream. The preponderance of star particles populate the trailing stream rather than the leading stream. The enhanced rotation speed of the satellite due to the fifth force means that the outward centrifugal

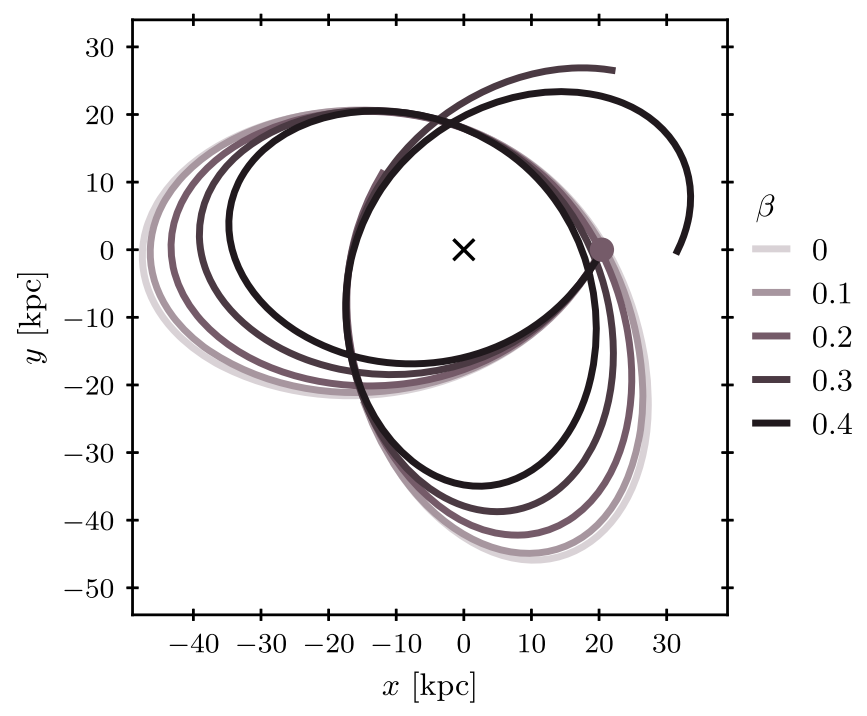

FIG. 5. Satellite B's orbit in its orbital plane, shown for a range of $\beta$ with $r_{\text {scr,sat }}=r_{\text {scr,Mw }}=0$. The cross indicates the Galactic center and the filled circle shows the final position of the satellite, i.e., the current observed position of the Sagittarius dwarf galaxy. This figure illustrates the effect of an unscreened fifth force on orbital shapes for a fixed final position. acceleration of the stars outweighs the inward gravitational acceleration by the Milky Way. Consequently, stars are more likely to leave the satellite via the outer Lagrange point. Also, even some of the stars which are disrupted from the inner Lagrange point can eventually end up in the trailing stream, once sufficient time has passed for them to be overtaken by the satellite. Meanwhile, the dark matter particles experience the same fifth force as the satellite, and so there is (almost) no preferential disruption via either Lagrange point. The dark matter stream that forms is consequently almost symmetric around the progenitor.

These effects are also apparent in Fig. 7, which shows the longitude difference $\Delta \Lambda=\Lambda-\Lambda_{\text {sat }}$ as a function of time for random subsamples of particles in the simulations without screening, with $\beta$ increasing in strength from 0.0 to 0.4 in steps of 0.1 for all four satellites. Here, $\Lambda$ is the longitude in the instantaneous orbital plane of the satellite and increases in the direction of the satellite's motion, so particles in the leading stream have positive $\Delta \Lambda$. The dark matter particles are stripped almost equally into the leading and trailing streams, leading to streams that are nearly symmetric about the progenitor for all values of $\beta$. For the star however, as $\beta$ increases, the particles are increasingly disrupted into negative longitudes, i.e., the trailing streams.

Sometimes, the satellite can be stripped completely of all of its stars. Then, the spatial separation between satellite and stream can be very large indeed, as no new stars become unbound from the satellite in order to bridge the gap. This occurs in satellite A for both $\beta=0.3$ and 0.4 , as it loses all of its stars at its first pericentric passage. Satellite A, which is significantly less massive than our other satellites, does not have a sufficiently deep potential well for its stars to remain bound under the enhanced centrifugal force from the Milky Way. Some caution is needed because assumption 3 for example (the assumption of an unchanging satellite mass and potential) may begin to break down when the disruption of the satellite due to the Milky Way is so severe. However, all of our satellites are, by assumption, dark matter dominated. Even in the simulations where the satellites lose all of their stars, they still retain a large fraction of their dark matter particles and, thus, most of their assumed mass.

This result echoes a key finding of Keselman, Nusser, and Peebles [15], who argued that this prediction of stellar streams without associated progenitors could be related to the observed orphan streams of the Milky Way.

\section{Chameleon screening}

We now show results from the chameleon simulations, i.e., the simulations with screening. Unlike the dark matter force investigated in the previous subsection, the fifth force here is universally coupled. However, as discussed in Secs. I and II, an effective EP violation arises because main sequence stars are self-screened against the fifth force in parameter regimes of interest. 


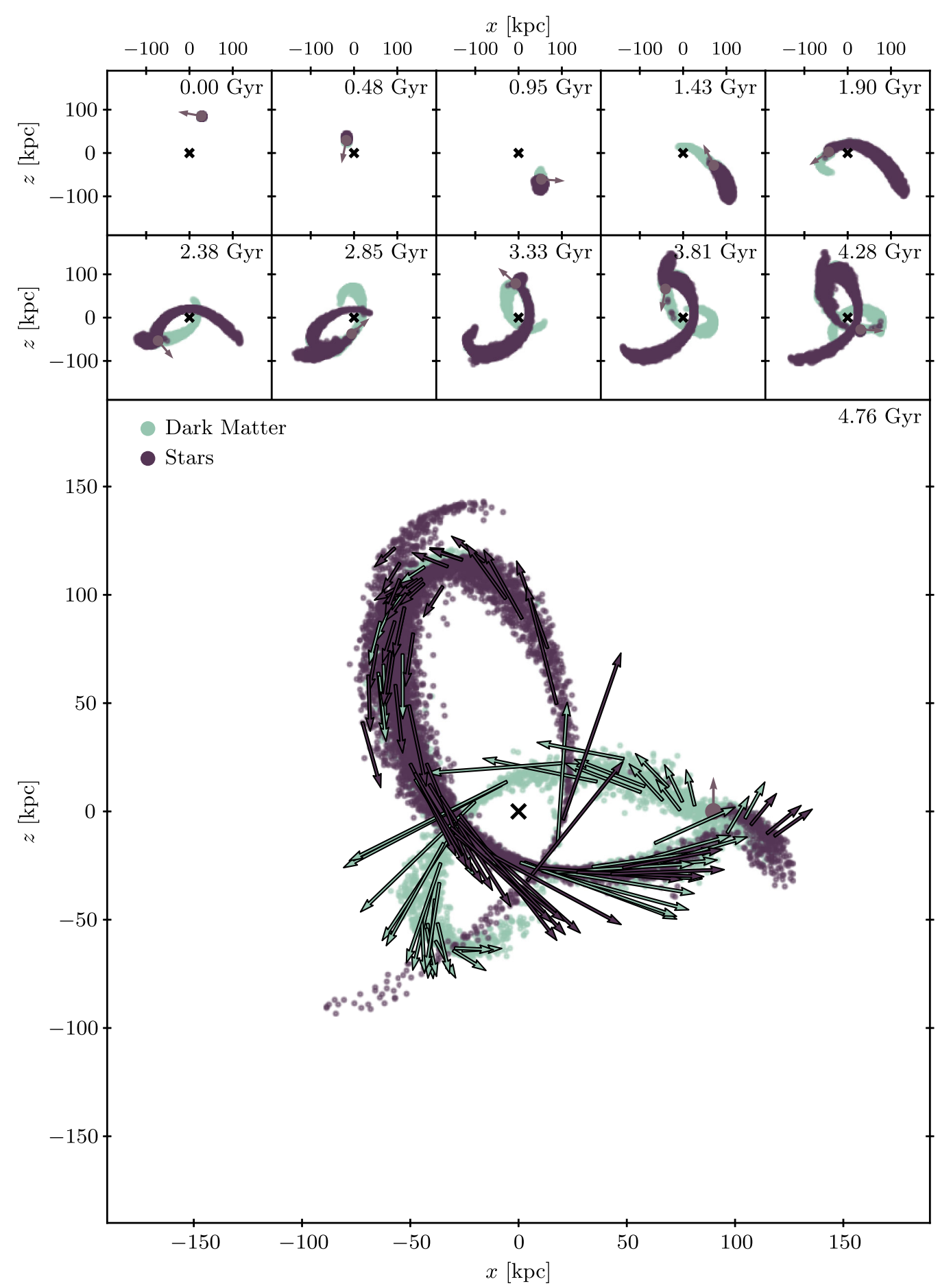

FIG. 6. The simulation depicted here is satellite $\mathrm{C}$ with no screening and a fifth force coupling only to dark matter with $\beta=0.2$. The large panel shows an image of the stellar (purple) and dark matter (green) streams at the end of the simulation, while the smaller panels above show the evolution over time. The interval between images is $1.5 \times 10^{16} \mathrm{~s}(\sim 0.48 \mathrm{Gyr}$, as labeled $)$. The cross and large filled circle, respectively, indicate the positions of the Milky Way and satellite centers. In the large panel, 50 unbound particles have been randomly chosen from each species, and arrows of the corresponding color are shown indicating their velocities. An animation of this simulation is included in Supplemental Material [51] accompanying this article. This figure shows the formation of an asymmetric stellar stream over time.

Figure 8 is the analog of Fig. 5, now showing the effect on the satellite's orbit of a varying Milky Way screening radius. In the case of the outermost screening radius of
$45 \mathrm{kpc}$, nearly the entire orbit is situated within $r_{\text {scr,Mw }}$ and is therefore almost equivalent to the standard gravity case. Following along this orbit from plotted position of the 

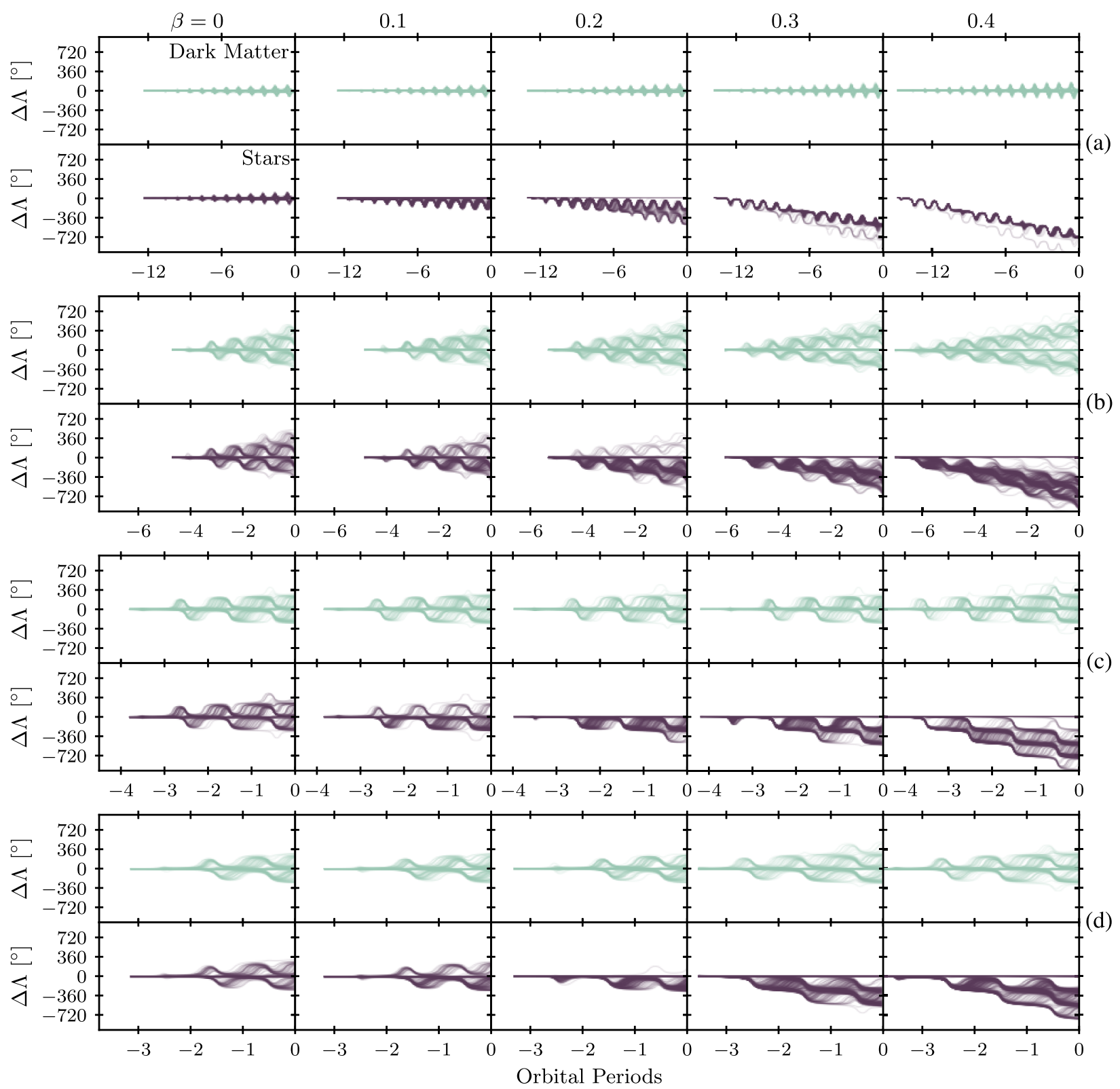

FIG. 7. The longitude difference $\Delta \Lambda=\Lambda-\Lambda_{\text {sat }}$ as a function of time for all four satellites without screening. Each column shows a different fifth force coupling from $\beta=0$ to 0.4 in steps of 0.1 . Here, $\Lambda$ is longitude in the orbital plane of the satellite, increasing in the direction of the satellite's orbit. Lines are drawn for 500 star and 500 DM particles in each simulation, i.e., one in 200 particles are randomly sampled. Complementing Fig. 6, this figure shows the development over time of the asymmetry of the stellar streams and the increased magnitude of this effect with $\beta$.

progenitor, the other orbits peel away one by one, in order of increasing screening radius. In other words, once the orbit passes outside the screening radius, the fifth force becomes active and the orbit starts to diverge from the standard gravity case. Recalling from Eq. (1) that the fifth force is proportional to the mass between the test particle and the screening radius, the divergences do not become noticeable as soon as the orbit passes out of a given screening radius, but some time after, once this enclosed mass is large enough for an appreciable fifth force.
Looking instead at the impact of the Milky Way screening radius on stream asymmetries, one observable quantity is the ratio of the number of stars in the leading to the trailing stream:

$$
\alpha=\frac{N_{\text {lead }}}{N_{\text {trail }}} .
$$

Figure 9 shows this quantity as a function of Milky Way screening radius for all satellites, assuming $Q_{\text {sat }}=1$, i.e., 


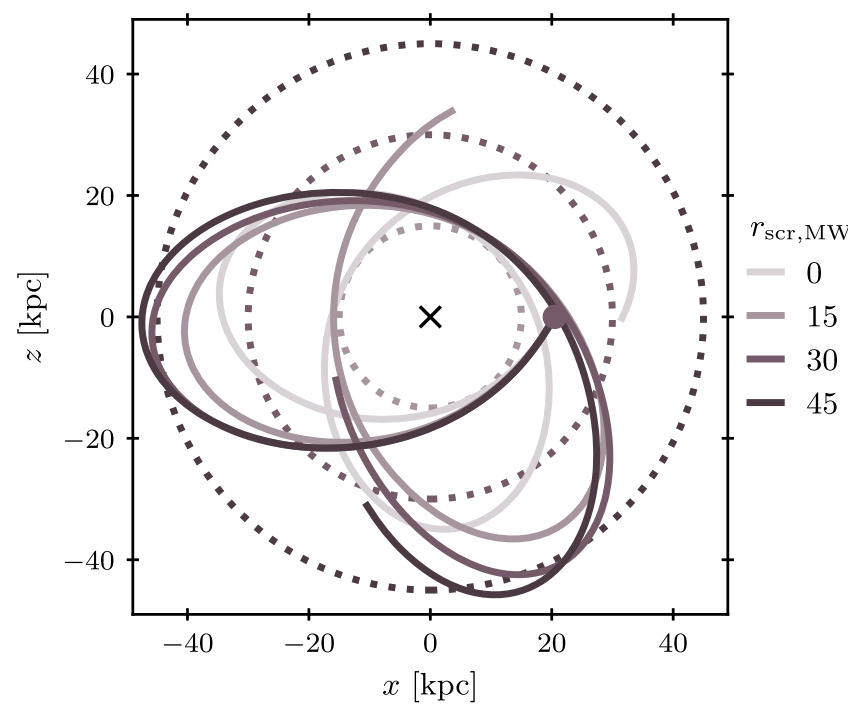

FIG. 8. Satellite B's orbit in its orbital plane, shown for $r_{\mathrm{scr}, \mathrm{MW}}=0,8,24,50,85 \mathrm{kpc}$, and $r_{\mathrm{scr}, \mathrm{sat}}=0, \beta=0.4$. The dotted circles indicate the position of the screening radius in each case. The cross indicates the Galactic center and the filled circle shows the final position of the satellite, i.e., the current observed position of the Sagittarius dwarf galaxy. This figure illustrates the effect of a Milky Way screening radius on the satellite orbital shapes.

fully unscreened satellites. To ensure a fair comparison between simulations, $\alpha$ is computed in each case at the moment of the satellite's third pericentric passage. As the MW screening radius increases, the asymmetry is progressively reduced. This appears to particularly be the case when $r_{\text {scr,Mw }}$ lies between the pericenter and apocenter of the orbit. This makes sense, as most tidal disruption occurs at and around pericentric passage. Therefore, screening the pericenter has the consequence of reducing the asymmetry of this disruption process. For all of our satellites, the streams are indistinguishable from those in the standard gravity case once $r_{\text {scr,Mw }}$ exceeds the apocentric distance.

We have observed in our simulations interesting signatures of chameleon gravity other than the stellar asymmetry. Examples of these are depicted in Fig. 10. First, in the extreme (high $\beta$ ) fifth force regime, the orbital paths of released stars around the Milky Way differ appreciably from their progenitor. However, because stars are released from the progenitor at different times, this also means that the liberated stars can be on different Milky Way orbits from each other. If most releases occur at pericentric passages, this can lead to a "striping" effect, with neighboring undulations of stars on the sky, corresponding to streams of stars released at successive pericentric passages. This effect is visible in the upper panel of Fig. 10.

Secondly, if the satellite itself is fully screened or almost so (i.e., low $Q_{\text {sat }}$ ), then it orbits the Milky Way more slowly than the dark matter that has been released and inhabits unscreened space. Then, we observe the opposite

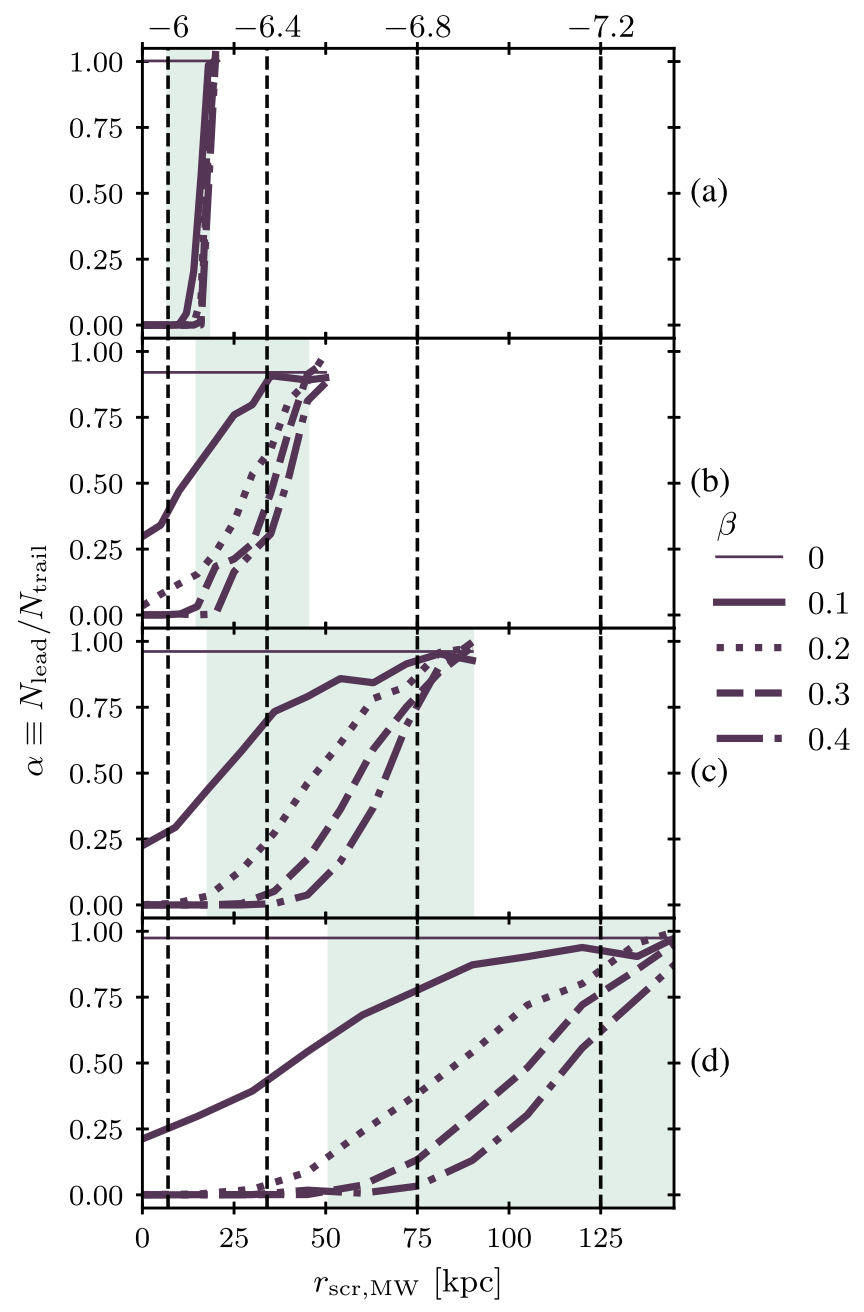

FIG. 9. The asymmetry parameter $\alpha \equiv N_{\text {lead }} / N_{\text {trail }}$, for all simulations with $Q_{\text {sat }}=1$. The four panels correspond to the four satellites and the different textures of line correspond to different values of $\beta$. In each panel, the shaded region indicates the radial range of the satellite's orbit. As with the horizontal lines in Fig. 2, the vertical dashed lines here show the locations of Milky Way screening radii for various values of $\log _{10}\left|f_{R 0}\right|$. This figure shows the Milky Way screening radius can affect the stream asymmetry. Streams at larger Galactocentric distances are sensitive to larger screening radii and, therefore, weaker modified gravity regimes.

asymmetry to that of the stars: the dark matter is preferentially disrupted into the leading stream rather than the trailing stream. This effect is shown in the lower panel of Fig. 10. While interesting, this effect is of course not readily accessible to observations.

\section{Future constraints}

The later Gaia data releases will likely enable the discovery of stellar streams at large distances from the Galactic center. As shown in Fig. 9, such streams are able to probe larger Milky Way screening radii and therefore 


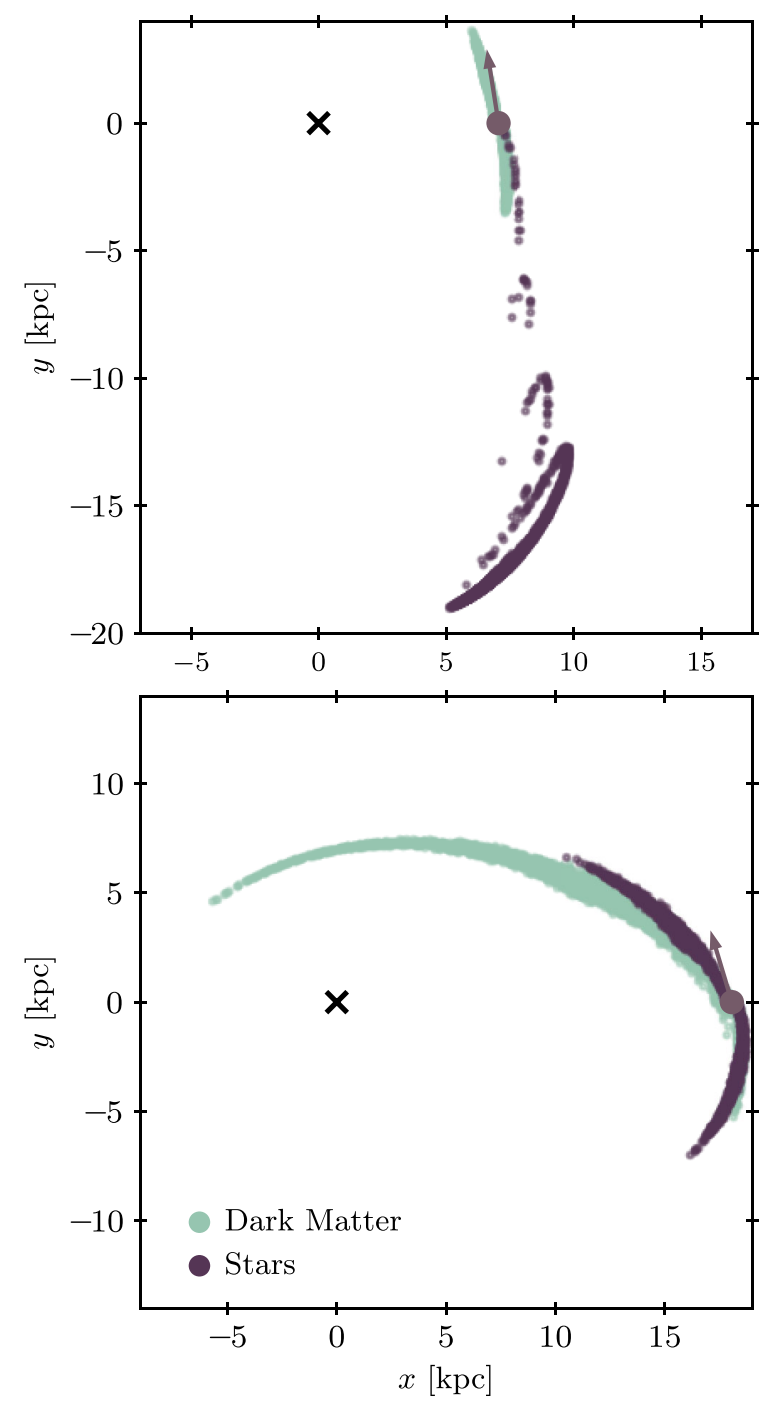

FIG. 10. Top: An image from a simulation of satellite A, $\beta=0.4, Q_{\text {sat }}=1$, and $r_{\text {scr,Mw }}=10 \mathrm{kpc}$. Bottom: An image from another simulation of satellite $\mathrm{A}, \beta=0.1, Q_{\text {sat }}=0$, and $r_{\text {scr,Mw }}=4 \mathrm{kpc}$. Animations of the two simulations depicted in this figure are included in Supplemental Material accompanying this article [51]. This figure shows some interesting signatures of screened modified gravity other than the stellar asymmetry we have discussed in previous figures.

"weaker," or more screened, regions of modified gravity parameter space.

Figure 11 shows $\alpha$ evaluated for all of our simulations of satellite $\mathrm{D}$, as a function of $r_{\mathrm{scr}, \mathrm{MW}}, Q_{\text {sat }}$, and $\beta$. As with Fig. 9, $\alpha$ is computed in each simulation at the moment of the satellite's third pericentric passage. This figure illustrates many of our earlier points; increasing $\beta$ increases the magnitude of the asymmetry, but the asymmetry is reduced by increasing $r_{\text {scr,sat }}$ (reducing $Q_{\text {sat }}$ ) or $r_{\text {scr,Mw }}$. In the $\beta=$ 0.4 case, approximately comparable to $f(R)$ gravity, the asymmetries grow large when $r_{\text {scr.MW }} \lesssim 100 \mathrm{kpc}$, assuming the satellite is fully unscreened $\left(Q_{\text {sat }}=1\right)$. Notably, this lies between the apocenter and pericenter of the satellite's orbit. Most tidal disruption occurs at pericentric passage, but here there is still enough disruption outside the screening radius, and sufficient numbers of leading stars lagging behind the satellite, that a large asymmetry develops anyway.

We can again use Hu-Sawicki $f(R)$ gravity to give an indication of the kinds of constraints attainable here. Figure 12 shows how the Milky Way screening radius depends on the parameter $f_{R 0}$. These calculations were performed using the scalar field solver within the $f(R)$ $N$-body code MG-Gadget [56]. MG-Gadget uses a NewtonGauss-Seidel relaxation method to solve the $f(R)$ equations of motion, calculating the scalar fields and fifth forces everywhere across a given mass distribution or within a given simulation volume. Such methods were first explored in the work of Oyaizu [59], and the subsequent years have seen a proliferation of codes simulating a myriad of modified gravity cosmologies [60-67].

Given a mass model for the Galaxy, this can then be sampled with a large number of particles, which are in turn fed to MG-Gadget to calculate the scalar field profile-and thus the Galaxy screening radius - for a given value of $f_{R 0}$. In each case, we truncate the mass model at $r_{100}$, the radius encompassing a region with density 100 times the cosmic critical density. In the case of the Milky Way, this radius has been shown to lie close to the "splashback radius," a reasonable definition for the edge of the Galaxy's halo [68].

The solid purple line in Fig. 12 shows the screening radii for the fiducial Milky Way model described in Sec. III A, i.e., the model used throughout our simulations. This illustrates the sensitivity of stream asymmetries as a probe of chameleon gravity.

However, the overall mass of the Milky Way is highly uncertain, and the primary source of uncertainty is the dark matter halo, particularly in its outer regions [69]. Thus, it is interesting to explore how this predicted sensitivity depends on the overall mass of the Galaxy. The radius $r_{100}$ for our fiducial model is $\sim 300 \mathrm{kpc}$, and the total mass $M_{100}$ (including baryons) enclosed within this radius is approximately $1.5 \times 10^{12} M_{\odot}$. Figure 12 additionally shows screening radii calculated for less massive (dotted) and more massive (dashed) Milky Way models. In these models the scale density $\rho_{0}$ of the dark matter halo has been rescaled by factors of 0.75 and 1.25 , respectively. These rescalings still lead to sensible values for the halo concentration and correspond to masses of $M_{100}=1.1$ and $2.0 \times 10^{12} M_{\odot}$ (note the overall masses are not rescaled by exactly 0.75 and 1.25 , because $r_{100}$ changes along with the density normalization). As expected, at fixed $f_{R 0}$, increasing (decreasing) the mass leads to an expansion (reduction) of the screening radius. These masses bracket a large range of reasonable estimates for the Milky Way mass, and so the region of the figure enclosed by these lines should in principle include the "true" screening radius of the Milky Way in an $f(R)$ universe. 


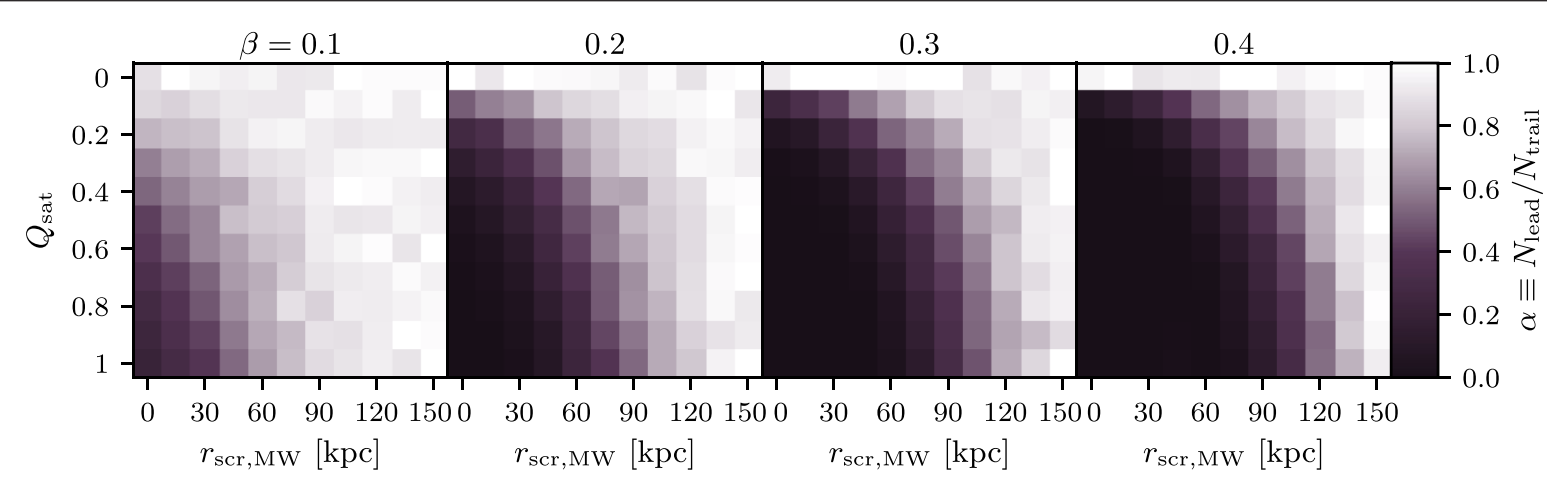

FIG. 11. The asymmetry parameter $\alpha \equiv N_{\text {lead }} / N_{\text {trail }}$ for the unbound stellar particles in all simulations of satellite D with screening,

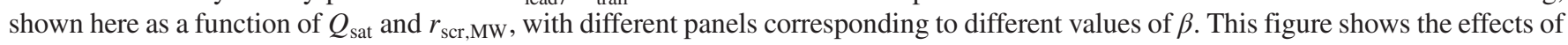
varying all of our parameters on the stream asymmetries.

One additional caveat, however, is that this treatment has ignored the environmental contributions to the scalar field by the Local Group. As a first approximation of this effect, the green lines in Fig. 12 show the Milky Way screening radii when a mass distribution for M31 is added to the MG-Gadget input. The model used for M31 is identical to our fiducial Milky Way model, centered at $(-380,620,-280) \mathrm{kpc}$ in Galactocentric coordinates [70]. There is a systematic upward shift in the Milky Way screening radii at all $f_{R 0}$ values except at $\log _{10}\left|f_{R 0}\right| \lesssim-8.5$, where $r_{\mathrm{MW}-\mathrm{M} 31}>\lambda_{C}$.

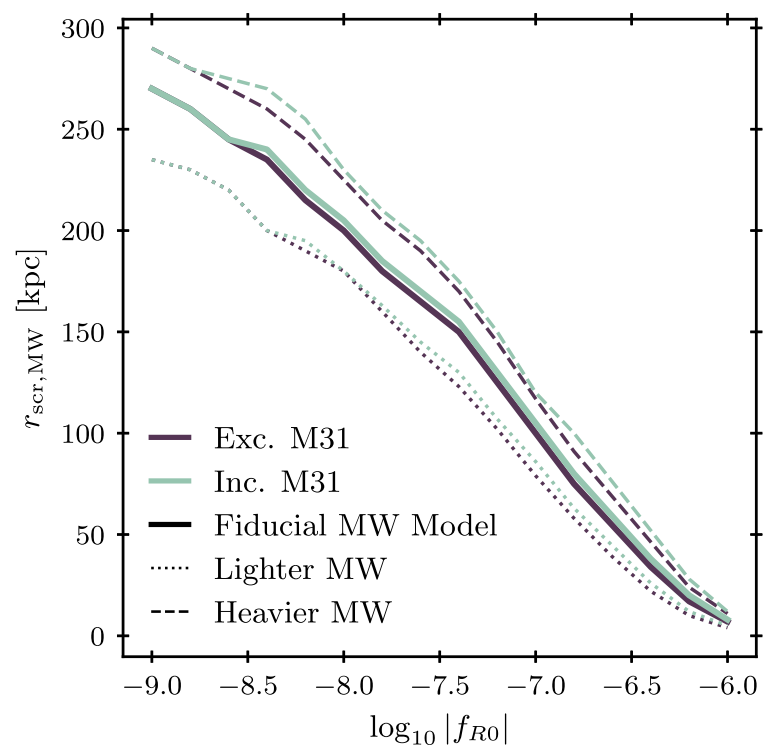

FIG. 12. The disk-plane Milky Way screening radius as a function of $\log _{10}\left|f_{R 0}\right|$. The solid lines show screening radii for the "fiducial" Milky Way model, i.e., the model described in Sec. III A. Meanwhile, the dotted and dashed lines represent Galaxy models in which the scale density $\rho_{0}$ of the dark matter halo has been rescaled by factors of 0.75 and 1.25 , respectively. The colors of the lines indicate the environment around the Galaxy, where violet lines are for an isolated Milky Way model, while the green lines additionally incorporate the contribution of M31. In every case, the screening radius is calculated with MG-Gadget.
However, the magnitude of this shift is typically rather small, on the order of a few kiloparsecs. This may increase with a more sophisticated model of the Local Group incorporating M33 and various other smaller galaxies, as well as the smooth intervening mass distribution. However, as the Milky Way and M31 are by far the most massive members of the Local Group, it seems likely that environmental screening will remain a subdominant effect.

We see from Fig. 12 that satellite D is able to probe the region $\log _{10}\left|f_{R 0}\right| \gtrsim-7.2$. However, if the satellite itself is partially screened, the sensitivity is greatly reduced. It is natural therefore to wonder about the degree to which a satellite would be screened at these values of $f_{R 0}$ and this region of the Milky Way's halo.

Figure 13 shows the scalar field profile around the Milky Way for $f_{R 0}=-10^{-7}$, again inferred using MGGadget. A Hernquist sphere identical to satellite $\mathrm{D}$ has been inserted at Galactocentric $(X=100, Y=0, Z=100) \mathrm{kpc}$. There is a clear screened region in the center of the Milky Way halo, with $r_{\text {scr,Mw }} \approx 100 \mathrm{kpc}$. The satellite is also partially screened, with a screened region of $r_{\text {scr,sat }} \approx$ $0.6 \mathrm{kpc}$ at its center. This corresponds to $Q_{\text {sat }}=0.8$. Comparing to Fig. 11, the suggestion is that, in an $f(R)$ universe, this satellite would provide very asymmetric streams. This is demonstrated in Fig. 14, which shows a simulation with a similar setup: satellite $\mathrm{D}$ with $\beta=0.4$, $r_{\text {scr,MW }}=105 \mathrm{kpc}$, and $Q_{\text {sat }}=0.8$. The left-hand panel shows the stream, while the right panel shows a more sophisticated observable signature than the asymmetry parameter: the cumulative number function of stars in each stream as a function of longitude in the orbital plane of the satellite. The difference in the two curves is rather striking and should be clearly discernible in the data.

The examples shown in Figs. 13 and 14 serve as proof of concept, demonstrating that stellar streams in the outer reaches of our Galaxy's halo are a sensitive probe of modified gravity. The observation of highly symmetric streams at large Galactocentric distances would rule out sizable fifth forces that couple differently to dark matter and stars in the outskirts of the Milky Way. This in turn would 


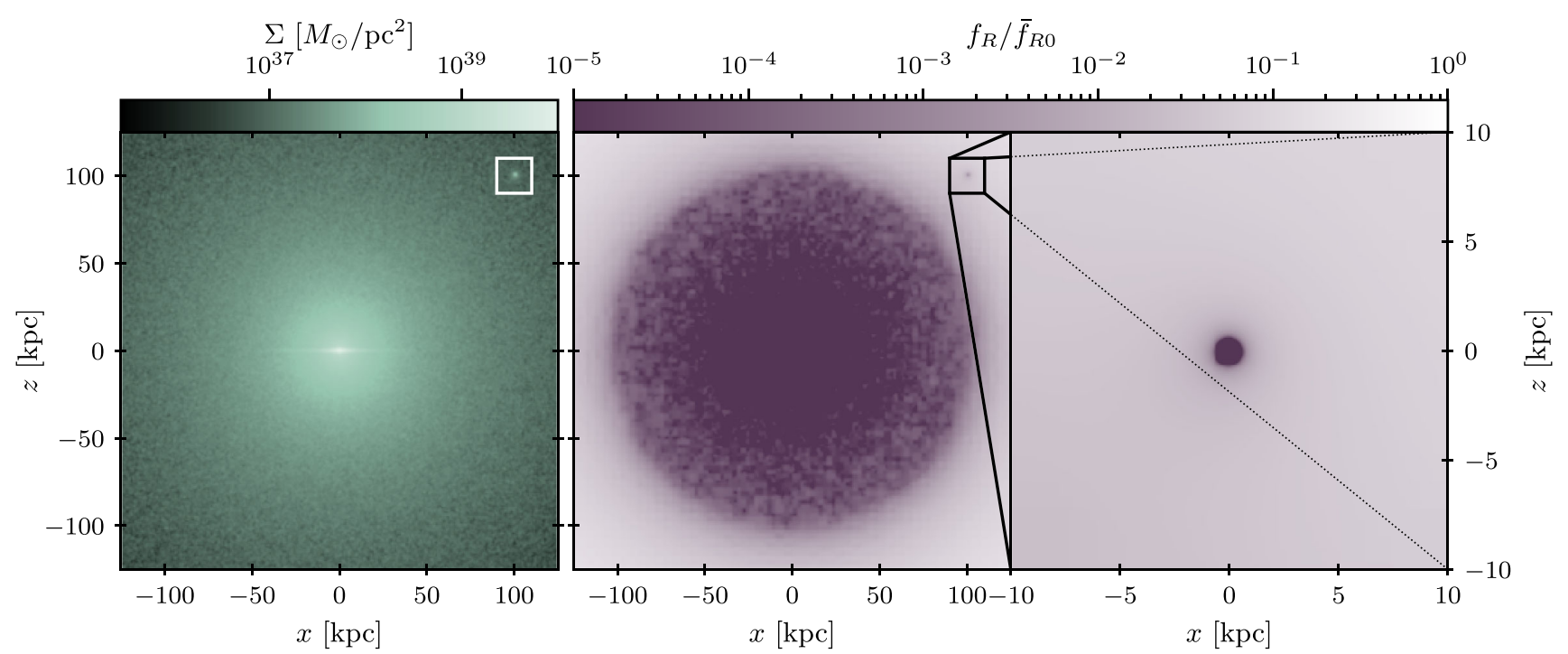

FIG. 13. Left: Edge-on particle density of Galaxy+satellite system fed to MG-Gadget to calculate the scalar field profile. The location of the satellite is indicated by the inset box. Middle: Scalar field profile for $f_{R 0}=-10^{-7}$ across the same system. The Milky Way's screened region is clearly discernible, while the satellite also has a small central screened region, shown in the right-hand panel, which shows a magnified image of the scalar field profile in a $20 \mathrm{kpc}$ region centered around the satellite.

provide sensitive constraints on screened modified gravity theories. For instance, looking at Fig. 12, symmetric streams at distances of $\sim 150-300 \mathrm{kpc}$ would require $\left|f_{R 0}\right| \sim 10^{-7.5}$ or even $10^{-8}$ to avoid sizable fifth forces in that radial range. This would be among the tightest constraints on $f(R)$ gravity achievable to date. However, we caution that environmental screening of the satellite may play a more significant role at these levels, but Fig. 11 suggests that only if the satellite is fully screened does the signal disappear entirely. Even when $Q_{\text {sat }}=0.1$, i.e., $90 \%$
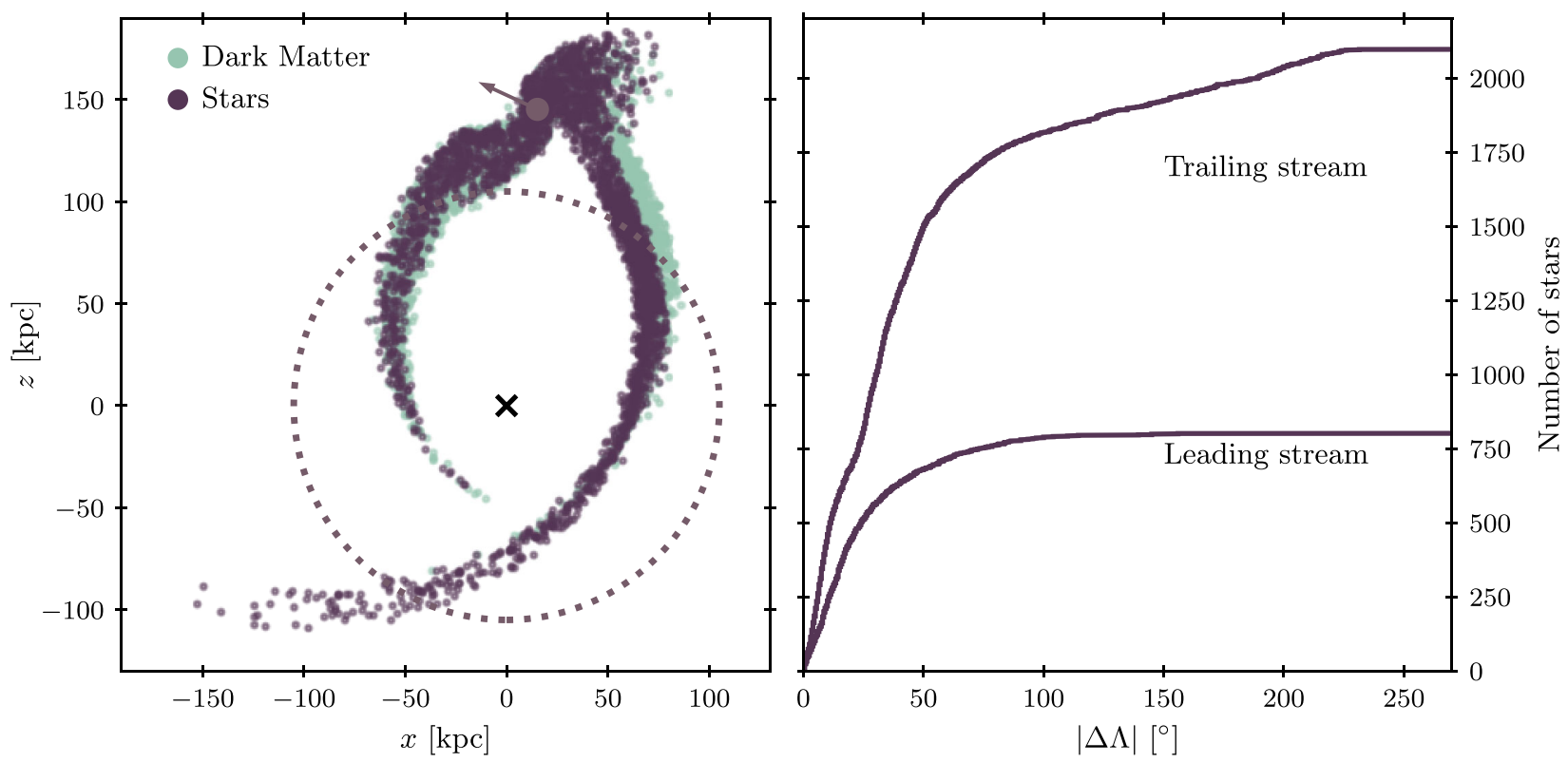

FIG. 14. Left: An image of a simulation of satellite $\mathrm{D}$, with $r_{\mathrm{scr}, \mathrm{MW}}=105 \mathrm{kpc}, Q_{\mathrm{sat}}=0.8$, and $\beta=0.4$. The dotted circle shows the location of the Milky Way screening radius, while the cross and filled circle show the locations of the Milky Way and satellite centers, respectively. The arrow shows the current direction of motion of the satellite. Right: Cumulative number of stars in either stream, as a function of longitude in the instantaneous orbital plane of the satellite. An animation of this simulation is included in Supplemental Material accompanying this article [51]. This figure, taken together with Fig. 13, shows that $f(R)$ gravity with $f_{R 0} \sim-10^{-7}$ should give a clear observational signature in stellar streams between 100 and $200 \mathrm{kpc}$. 
of the mass is screened, there is still an appreciable signal. So, given the observation of a large number of symmetric streams and if there is little environmental screening by the Local Group, then constraints down to these levels are feasible.

On the other hand, observations of highly asymmetric streams would strengthen the case for screened modified gravity theories. It should be noted, however, that mild asymmetries can arise due to dynamical effects. Indeed, an asymmetry between the leading and trailing streams is expected from Eq. (27). This may be compounded by dynamical interactions with dark subhaloes or other satellites [71], asymmetries in the stellar populations in the progenitor satellite [72,73], effects of the Galactic bar $[54,74,75]$ and regions of chaos in the Galactic potential [76]. Such effects would have to be carefully weighed before a modified gravity interpretation could be seriously considered for such observations.

\section{CONCLUSIONS}

We have investigated possible imprints of chameleon gravity on stellar streams from dwarf galaxies around the Milky Way. While canonical chameleon theories are universally coupled, an effective violation of the EP arises because of the self-screening of main sequence stars, as noted by Hui, Nicolis, and Stubbs [34]. Consequently, stars are preferentially stripped from the progenitor into the trailing stream rather than the leading stream.

We have created a restricted $N$-body code (smoggy; made publicly available [77]) and used it to simulate the formation of tidal streams from progenitors with a variety of masses and Galactocentric distances. We considered a range of modified gravity scenarios (coupling strength, Milky Way screening level, satellite screening level) in each case.

As found by Kesden and Kamionkowski [13,14], an EPviolating fifth force that couples to dark matter but not baryons causes asymmetries to develop in stellar streams with dark-matter-dominated progenitors. The stars are preferentially disrupted via the outer Lagrange points into the trailing streams. We have corrected and augmented the analytic calculations of Ref. [13] for point masses so that they are also applicable to extended Galactic mass distributions like isothermal spheres. The effect of these changes is to make the test more sensitive to EP-violating fifth forces. For the most massive dwarf spheroidals, like Sagittarius or Fornax, the criterion given in Eq. (29) suggests values of $\beta^{2} \gtrsim 10^{-3}$ can be probed. For the smallest dwarf spheroidals such as Segue 1 with a mass of $6 \times 10^{5} M_{\odot}$, then values of $\beta^{2} \gtrsim 10^{-4}$ are in principle accessible. As a rule of thumb for a satellite with mass $m$ at a location enclosing a Milky Way mass $M$, the form of the criterion suitable for a flat rotation curve galaxy is

$$
\beta^{2} \gtrsim 2^{-5 / 3}\left(\frac{m}{M}\right)^{2 / 3}
$$

This asymmetry also occurs in the chameleon context, when screening radii are introduced to the Milky Way and satellite, and with stars self-screening. The magnitude of the asymmetry depends on the coupling strength $\beta$, the Milky Way screening radius, as well as the degree of screening of the stream progenitor; large values of $\beta$ give large asymmetries, but these are reduced with increasing $r_{\text {scr, MW }}$ and $r_{\text {scr,sat }}$.

Our simulations - the most comprehensive to date for the formation of tidal streams under chameleon gravityhave revealed further interesting effects. First, the trailing stellar stream may become detached from the dark matter progenitor if all the stars are exhausted by earlier pericentric stripping (cf. [15]). As an example, this effect is visible in Fig. 7 and occurs for low mass satellites in the extreme fifth force regime. Second, prominent striations in the stellar trailing tail may exist if stars are stripped at repeated pericentric passages by a strong fifth force. Thirdly, if the satellite is fully screened, its orbital frequency is lower than that of its associated dark matter. This leads to strong asymmetries in the dark matter distribution, which is preferentially liberated into the leading tidal tail.

Taking Hu-Sawicki $f(R)$ gravity with $f_{R 0}=-10^{-7}$ as an example, we derive a Milky Way screening radius of around $100 \mathrm{kpc}$. A massive dwarf spheroidal galaxy at a distance of $\approx 150 \mathrm{kpc}$ - such as Fornax — would be partially screened but would nonetheless produce highly asymmetric streams under tidal disruption.

The ratio of the cumulative number function of stars in the leading and trailing stream as a function of longitude from the satellite is computable from simulations and measurable from the observational data and can provide a direct test of theories with screening mechanisms like chameleon gravity. The later Gaia data releases may lead to discoveries of stellar streams at distances $\gtrsim 100 \mathrm{kpc}$ from the Galactic center. These streams will be a sensitive probe of modified gravity; such highly asymmetric streams at these distances would be telltale signatures of modified gravity.

On the other hand, if the data uncover a number of very symmetric streams, then constraints down to the level of $f_{R 0} \sim-10^{-8}$ - the tightest constraints to date-could be attainable if the screening of the satellite and other nuisance parameters are carefully taken into account. Also, our assumption that the Compton wavelength is much larger than relevant length scales begins to break down at such values of $f_{R 0}$, and Yukawa suppression will become appreciable below $f_{R 0} \sim-10^{-8}$. Of course, the investigation need not be limited to Hu-Sawicki $f(R)$ gravity. Sensitive constraints will also be attainable in the general 
chameleon parameter space, and we merely use $f(R)$ gravity as a fiducial theory.

This desirability of streams at large distances suggests another interesting avenue for exploration: stellar streams around other galaxies. Streams have already been observed around other galaxies (e.g., [78,79]), and it seems likely that future wide-field surveys such as LSST [80] will uncover more streams at large distances from their host galaxies. This, combined with a calculation of the host galaxy screening properties (e.g., via the screening maps of Ref. [81]) could also be a sensitive probe of screened modified gravity.

Finally, we note that other screened modified gravity theories can also be probed with stellar streams. For instance, the symmetron screening mechanism [82,83] has a simple density threshold as a screening criterion. Consequently, there will necessarily be a region of parameter space in which the stars are screened, but the surrounding diffuse dark matter component is not. In this regime, stream asymmetries will also be present and are worthy of future investigation.

\section{ACKNOWLEDGMENTS}

The authors would like to acknowledge Matt Auger, Vasily Belokurov, Sergey Koposov, Jason Sanders, and Denis Erkal for helpful discussions and the anonymous referee for reviewing and suggesting various improvements to the manuscript. A.P.N. thanks the Science and Technology Facilities Council (STFC) for their Ph.D. studentship. H.Z. acknowledges support by the Kavli Foundation. A.-C.D. acknowledges partial support from STFC under Grants No. ST/L000385 and No. ST/L000636. This work used the DiRAC Data Analytic system at the University of Cambridge, operated by the University of Cambridge High Performance Computing Service on behalf of the STFC DiRAC HPC Facility. This equipment was funded by BIS National E-infrastructure capital grant (ST/K001590/1), STFC capital Grants No. ST/H008861/1 and No. ST/H00887X/1, and STFC DiRAC Operations Grant No. ST/K00333X/1. DiRAC is part of the National E-Infrastructure.
[1] V. Belokurov et al., The field of streams: Sagittarius and its siblings, Astrophys. J. 642, L137 (2006).

[2] K. V. Johnston, A prescription for building the Milky Way's halo from disrupted satellites, Astrophys. J. 495, 297 (1998).

[3] T. Prusti et al. (Gaia Collaboration), The Gaia mission, Astron. Astrophys. 595, A1 (2016).

[4] A. G. A. Brown et al. (Gaia Collaboration), Gaia Data Release 2. Summary of the contents and survey properties, Astron. Astrophys. 616, A1 (2018).

[5] G. C. Myeong, N. W. Evans, V. Belokurov, N. C. Amorisco, and S. E. Koposov, Halo substructure in the SDSS-Gaia catalogue: Streams and clumps, Mon. Not. R. Astron. Soc. 475, 1537 (2018).

[6] K. Malhan, R. A. Ibata, and N. F. Martin, Ghostly tributaries to the Milky Way: Charting the Halo's stellar streams with the Gaia DR2 catalogue, Mon. Not. R. Astron. Soc. 481, 3442 (2018).

[7] S. Meingast, J. Alves, and V. Fürnkranz, Extended stellar systems in the solar neighborhood. II. Discovery of a nearby $120^{\circ}$ stellar stream in Gaia DR2, Astron. Astrophys. 622, L13 (2019).

[8] S. E. Koposov et al. (Orphan Aspen Treasury Collaboration), Piercing the Milky Way: An all-sky view of the orphan stream, Mon. Not. R. Astron. Soc. 485, 4726 (2019).

[9] T. S. Li et al. ( $\mathrm{S}^{5}$ Collaboration), The southern stellar stream spectroscopic survey $\left(\mathrm{S}^{5}\right)$ : Overview, target selection, data reduction, validation, and early science, Mon. Not. R. Astron. Soc. 490, 3508 (2019).

[10] J. L. Sanders and J. Binney, Stream-orbit misalignment-I. The dangers of orbit-fitting, Mon. Not. R. Astron. Soc. 433, 1813 (2013).
[11] A. Bowden, V. Belokurov, and N. W. Evans, Dipping our toes in the water: First models of GD-1 as a stream, Mon. Not. R. Astron. Soc. 449, 1391 (2015).

[12] G. F. Thomas, B. Famaey, R. Ibata, F. Renaud, N. F. Martin, and P. Kroupa, Stellar streams as gravitational experiments. II. Asymmetric tails of globular cluster streams, Astron. Astrophys. 609, A44 (2018).

[13] M. Kesden and M. Kamionkowski, Tidal tails test the equivalence principle in the dark-matter sector, Phys. Rev. D 74, 083007 (2006).

[14] M. Kesden and M. Kamionkowski, Galilean Equivalence for Galactic Dark Matter, Phys. Rev. Lett. 97, 131303 (2006).

[15] J. A. Keselman, A. Nusser, and P. J. E. Peebles, Galaxy satellites and the weak equivalence principle, Phys. Rev. D 80, 063517 (2009).

[16] L. Amendola and S. Tsujikawa, Dark Energy: Theory and Observations (Cambridge University Press, Cambridge, England, 2010).

[17] T. Clifton, P. G. Ferreira, A. Padilla, and C. Skordis, Modified gravity and cosmology, Phys. Rep. 513, 1 (2012).

[18] A. Joyce, B. Jain, J. Khoury, and M. Trodden, Beyond the cosmological standard model, Phys. Rep. 568, 1 (2015).

[19] K. Koyama, Cosmological tests of modified gravity, Rep. Prog. Phys. 79, 046902 (2016).

[20] B. Jain and J. Khoury, Cosmological tests of gravity, Ann. Phys. (Amsterdam) 325, 1479 (2010).

[21] J. Khoury, Theories of dark energy with screening mechanisms, arXiv:1011.5909.

[22] J. Khoury and A. Weltman, Chameleon cosmology, Phys. Rev. D 69, 044026 (2004). 
[23] H. A. Buchdahl, Non-linear Lagrangians and cosmological theory, Mon. Not. R. Astron. Soc. 150, 1 (1970).

[24] W. Hu and I. Sawicki, Models of $f(R)$ cosmic acceleration that evade solar system tests, Phys. Rev. D 76, 064004 (2007).

[25] P. Brax, C. van de Bruck, A.-C. Davis, and D. J. Shaw, f(R) gravity and chameleon theories, Phys. Rev. D 78, 104021 (2008).

[26] C. Burrage and J. Sakstein, Tests of chameleon gravity, Living Rev. Relativity 21, 1 (2018).

[27] T. Baker et al., The novel probes project-Tests of gravity on astrophysical scales, arXiv:1908.03430.

[28] A. P. Naik, E. Puchwein, A.-C. Davis, and C. Arnold, Imprints of Chameleon $f(R)$ gravity on Galaxy rotation curves, Mon. Not. R. Astron. Soc. 480, 5211 (2018).

[29] A. P. Naik, E. Puchwein, A.-C. Davis, D. Sijacki, and H. Desmond, Constraints on chameleon $\mathrm{f}(\mathrm{R})$-gravity from galaxy rotation curves of the SPARC sample, Mon. Not. R. Astron. Soc. 489, 771 (2019).

[30] H. Desmond, P. G. Ferreira, G. Lavaux, and J. Jasche, Fifth force constraints from galaxy warps, Phys. Rev. D 98, 083010 (2018).

[31] H. Desmond, P. G. Ferreira, G. Lavaux, and J. Jasche, Fifth force constraints from the separation of galaxy mass components, Phys. Rev. D 98, 064015 (2018).

[32] H. Desmond, P. G. Ferreira, G. Lavaux, and J. Jasche, The fifth force in the local cosmic web, Mon. Not. R. Astron. Soc. 483, L64 (2019).

[33] V. Vikram, J. Sakstein, C. Davis, and A. Neil, Astrophysical tests of modified gravity: Stellar and gaseous rotation curves in dwarf galaxies, Phys. Rev. D 97, 104055 (2018).

[34] L. Hui, A. Nicolis, and C. W. Stubbs, Equivalence principle implications of modified gravity models, Phys. Rev. D 80, 104002 (2009).

[35] A.-C. Davis, E. A. Lim, J. Sakstein, and D. J. Shaw, Modified gravity makes galaxies brighter, Phys. Rev. D 85, 123006 (2012).

[36] J. Sakstein, Stellar oscillations in modified gravity, Phys. Rev. D 88, 124013 (2013).

[37] H. Goldstein, Classical Mechanics, 1st ed. (AddisonWesley, New York, 1951).

[38] J. Binney and S. Tremaine, Galactic Dynamics, 2nd ed. (Princeton University Press, Princeton, NJ, 2008).

[39] N. C. Amorisco and N. W. Evans, Phase-space models of the dwarf spheroidals, Mon. Not. R. Astron. Soc. 411, 2118 (2011).

[40] M. Niederste-Ostholt, V. Belokurov, N. W. Evans, G. Gilmore, R. F. G. Wyse, and J. E. Norris, The origin of Segue 1, Mon. Not. R. Astron. Soc. 398, 1771 (2009).

[41] P. J. McMillan, The mass distribution and gravitational potential of the Milky Way, Mon. Not. R. Astron. Soc. 465, 76 (2017).

[42] J. F. Navarro, C. S. Frenk, and S. D. M. White, A universal density profile from hierarchical clustering, Astrophys. J. 490, 493 (1997).

[43] M. Schaller, C. S. Frenk, R. G. Bower, T. Theuns, A. Jenkins, J. Schaye, R. A. Crain, M. Furlong, C. Dalla Vecchia, and I. G. McCarthy, Baryon effects on the internal structure of $\Lambda \mathrm{CDM}$ haloes in the EAGLE simulations, Mon. Not. R. Astron. Soc. 451, 1247 (2015).
[44] N. Bissantz and O. Gerhard, Spiral arms, bar shape and bulge microlensing in the Milky Way, Mon. Not. R. Astron. Soc. 330, 591 (2002).

[45] https://github.com/aneeshnaik/mw_poisson.

[46] W. Dehnen and J. Binney, Mass models of the Milky Way, Mon. Not. R. Astron. Soc. 294, 429 (1998).

[47] R. R. Lane, A. H. W. Küpper, and D. C. Heggie, The tidal tails of 47 Tucanae, Mon. Not. R. Astron. Soc. 423, 2845 (2012).

[48] A. H. W. Küpper, R. R. Lane, and D. C. Heggie, More on the structure of tidal tails, Mon. Not. R. Astron. Soc. 420, 2700 (2012).

[49] S. L. J. Gibbons, V. Belokurov, and N. W. Evans, 'Skinny Milky Way please', says Sagittarius, Mon. Not. R. Astron. Soc. 445, 3788 (2014).

[50] https://github.com/aneeshnaik/smoggy.

[51] See Supplemental Material at http://link.aps.org/ supplemental/10.1103/PhysRevD.102.084066 for animations of the simulations depicted in Figs. 4, 6, 10, and 14.

[52] L. Hernquist, An analytical model for spherical galaxies and bulges, Astrophys. J. 356, 359 (1990).

[53] N. W. Evans and J.H. An, Distribution function of dark matter, Phys. Rev. D 73, 023524 (2006).

[54] S. Pearson, A. M. Price-Whelan, and K. V. Johnston, Gaps and length asymmetry in the stellar stream Palomar 5 as effects of Galactic bar rotation, Nat. Astron. 1, 633 (2017).

[55] D. R. Law and S.R. Majewski, The Sagittarius dwarf galaxy: A model for evolution in a triaxial Milky Way Halo, Astrophys. J. 714, 229 (2010).

[56] E. Puchwein, M. Baldi, and V. Springel, Modified-gravityGADGET: A new code for cosmological hydrodynamical simulations of modified gravity models, Mon. Not. R. Astron. Soc. 436, 348 (2013).

[57] M. Boylan-Kolchin, C.-P. Ma, and E. Quataert, Dynamical friction and galaxy merging time-scales, Mon. Not. R. Astron. Soc. 383, 93 (2008).

[58] A. Cabré, V. Vikram, G.-B. Zhao, B. Jain, and K. Koyama, Astrophysical tests of gravity: A screening map of the nearby universe, J. Cosmol. Astropart. Phys. 07 (2012) 034.

[59] H. Oyaizu, Nonlinear evolution of $f(R)$ cosmologies. I. Methodology, Phys. Rev. D 78, 123523 (2008).

[60] B. Li and H. Zhao, Structure formation by a fifth force: N-body versus linear simulations, Phys. Rev. D 80, 044027 (2009).

[61] F. Schmidt, Self-consistent cosmological simulations of DGP braneworld gravity, Phys. Rev. D 80, 043001 (2009).

[62] H. Zhao, A. V. Macciò, B. Li, H. Hoekstra, and M. Feix, Structure formation by fifth force: Power spectrum from N-body simulations, J. Cosmol. Astropart. Phys. 02 (2010) L179.

[63] P. Brax, C. van de Bruck, A.-C. Davis, B. Li, and D. J. Shaw, Nonlinear structure formation with the environmentally dependent dilaton, Phys. Rev. D 83, 104026 (2011).

[64] P. Brax, A.-C. Davis, B. Li, H. A. Winther, and G.-B. Zhao, Systematic simulations of modified gravity: Symmetron and dilaton models, J. Cosmol. Astropart. Phys. 10 (2012) 002.

[65] B. Li, G.-B. Zhao, R. Teyssier, and K. Koyama, ECOSMOG: An Efficient COde for Simulating MOdified Gravity, J. Cosmol. Astropart. Phys. 01 (2012) 051. 
[66] B. Li, G.-B. Zhao, and K. Koyama, Exploring Vainshtein mechanism on adaptively refined meshes, J. Cosmol. Astropart. Phys. 05 (2013) 023.

[67] C. Llinares and D. F. Mota, Releasing Scalar Fields: Cosmological Simulations of Scalar-Tensor Theories for Gravity Beyond the Static Approximation, Phys. Rev. Lett. 110, 161101 (2013).

[68] A. J. Deason, A. Fattahi, C. S. Frenk, R. J. J. Grand, K. A. Oman, S. Garrison-Kimmel, C. M. Simpson, and J.F. Navarro, The edge of the Galaxy, Mon. Not. R. Astron. Soc. 496, 3929 (2020).

[69] J. Bland-Hawthorn and O. Gerhard, The galaxy in context: Structural, kinematic, and integrated properties, Annu. Rev. Astron. Astrophys. 54, 529 (2016).

[70] R. P. van der Marel, M. Fardal, G. Besla, R. L. Beaton, S. T. Sohn, J. Anderson, T. Brown, and P. Guhathakurta, The M31 velocity vector. II. Radial orbit toward the Milky Way and implied local group mass, Astrophys. J. 753, 8 (2012).

[71] D. Erkal and V. Belokurov, Forensics of subhalo-stream encounters: The three phases of gap growth, Mon. Not. R. Astron. Soc. 450, 1136 (2015).

[72] J. Peñarrubia, V. Belokurov, N. W. Evans, D. MartínezDelgado, G. Gilmore, M. Irwin, M. Niederste-Ostholt, and D. B. Zucker, Was the progenitor of the Sagittarius stream a disc galaxy?, Mon. Not. R. Astron. Soc. 408, L26 (2010).

[73] A. Bonaca, C. Conroy, A. M. Price-Whelan, and D. W. Hogg, Multiple components of the Jhelum stellar stream, J. Cosmol. Astropart. Phys. 02 (2019) L37.
[74] K. Hattori, D. Erkal, and J. L. Sanders, Shepherding tidal debris with the Galactic bar: The Ophiuchus stream, Mon. Not. R. Astron. Soc. 460, 497 (2016).

[75] D. Erkal, S. E. Koposov, and V. Belokurov, A sharper view of Pal 5's tails: Discovery of stream perturbations with a novel non-parametric technique, Mon. Not. R. Astron. Soc. 470, 60 (2017).

[76] A. M. Price-Whelan, B. Sesar, K. V. Johnston, and H.-W. Rix, Spending too much time at the Galactic bar: Chaotic fanning of the ophiuchus stream, Astrophys. J. 824, 104 (2016).

[77] https://github.com/aneeshnaik/smoggy.

[78] D. Martínez-Delgado, J. Peñarrubia, R. J. Gabany, I. Trujillo, S. R. Majewski, and M. Pohlen, The ghost of a dwarf Galaxy: Fossils of the hierarchical formation of the nearby spiral Galaxy NGC 5907, Astrophys. J. 689, 184 (2008).

[79] D. Martínez-Delgado et al., Stellar tidal streams in spiral galaxies of the local volume: A pilot survey with modest aperture telescopes, Astron. J. 140, 962 (2010).

[80] Ž. Ivezić et al., LSST: From science drivers to reference design and anticipated data products, Astrophys. J. 873, 111 (2019).

[81] H. Desmond, P. G. Ferreira, G. Lavaux, and J. Jasche, Reconstructing the gravitational field of the local Universe, Mon. Not. R. Astron. Soc. 474, 3152 (2018).

[82] K. Hinterbichler and J. Khoury, Screening Long-Range Forces through Local Symmetry Restoration, Phys. Rev. Lett. 104, 231301 (2010).

[83] K. Hinterbichler, J. Khoury, A. Levy, and A. Matas, Symmetron cosmology, Phys. Rev. D 84, 103521 (2011). 